\title{
A Robust Computational Algorithm for Inverse Photomask Synthesis in Optical Projection Lithography*
}

\author{
Siu Kai Choy ${ }^{\dagger}$, Ningning Jia ${ }^{\ddagger}$, Chong Sze Tong ${ }^{\S}$, Man Lai Tang ${ }^{\S}$, and Edmund Y. Lam ${ }^{\ddagger}$
}

\begin{abstract}
Inverse lithography technology formulates the photomask synthesis as an inverse mathematical problem. To solve this, we propose a variational functional and develop a robust computational algorithm, where the proposed functional takes into account the process variations and incorporates several regularization terms that can control the mask complexity. We establish the existence of the minimizer of the functional, and in order to optimize it effectively, we adopt an alternating minimization procedure with Chambolle's fast duality projection algorithm. Experimental results show that our proposed algorithm is effective in synthesizing high quality photomasks as compared with existing methods.
\end{abstract}

Key words. inverse lithography, image synthesis, total variation

AMS subject classifications. 65K10, 65R32, 68U10, 93B40

DOI. $10.1137 / 110830356$

1. Introduction. As a critical step in the semiconductor manufacturing process, optical lithography serves to imprint the circuit design on the photomask onto silicon wafers via light. It is known that deviation occurs on the printed image due to the subwavelength circuit size on the mask $[47,40]$. Such a deviation poses great challenges to the industry in terms of maintaining the printed pattern integrity of the original design. One of the widely adopted techniques to remedy the aforementioned difficulty is the so-called optical proximity correction (OPC) $[6,29,46]$, which is used to compensate for the imaging errors due to diffraction and process variations by predistorting the patterns on the template or the mask [47]. The predistortion can be guided by a set of rules implemented in the precomputed lookup tables to move the contours or by simulating the fabrication process using mathematical models to dynamically adjust the mask pattern. Based on the forward imaging model (as will be introduced in section 3), inverse lithography technology (ILT) has emerged as a promising approach to OPC that uses pixelated pattern images and computes the optimal mask by

\footnotetext{
${ }^{*}$ Received by the editors April 11, 2011; accepted for publication (in revised form) February 14, 2012; published electronically May 17, 2012.

http://www.siam.org/journals/siims/5-2/83035.html

${ }^{\dagger}$ Department of Mathematics and Statistics, Hang Seng Management College, Shatin, Hong Kong (skchoy@ hsmc.edu.hk).

${ }^{\ddagger}$ Imaging Systems Laboratory, Department of Electrical and Electronic Engineering, The University of Hong Kong, Pokfulam Road, Hong Kong (nnjia@eee.hku.hk, elam@eee.hku.hk). The research of these authors was supported in part by the University Research Committee of the University of Hong Kong under project 10400898, by the Research Grants Council of the Hong Kong Special Administrative Region, China, under projects HKU 7134/08E, and by the UGC Areas of Excellence project Theory, Modeling, and Simulation of Emerging Electronics.

${ }^{\S}$ Department of Mathematics, Hong Kong Baptist University, Kowloon Tong, Hong Kong (cstong@hkbu.edu.hk, mltang@hkbu.edu.hk). The research of these authors was supported by the RGC GRF under grants HKBU202108, HKBU261007, and HKBU261508.
} 
solving an inverse mathematical problem.

Equipped with increasingly sophisticated mathematical models of the lithography process, researchers have developed various computational schemes [18] which facilitate the adoption of optimization algorithms to solve the inverse mask design problem. From the earliest studies in the 1990s [21] until now, many algorithms have been developed for more robust solutions [30, 43, 35, 8, 42, 48, 24, 38, 23, 33, 15]. For instance, Sherif, Saleh, and De Leone [45] used branch and bounds methods to synthesize the mask. Liu and Zakhor [21] designed binary and phase-shifting masks based on simulated annealing. In [31], the authors adopted the method of projection on convex sets for phase-shifting mask synthesis. Granik [12] proposed a fast implementation scheme for solving an objective function which consists of linear, quadratic, and nonlinear terms. A genetic algorithm [9], nonlinear programming [11], and a randompixel flipping technique [28] have also been proposed for mask synthesis. Recently, Poonawala and Milanfar [35] formulated the mask synthesis problem by a continuous functional that comprises data fidelity, quadratic, and complexity penalty terms. The functional was then solved by the steepest descent algorithm. Since then, many efforts have been devoted to refining the implementation of gradient-based optimization $[4,34,36,22,3]$. Such extensive exploration has pushed ILT from theory to practice.

While the development of lithography technology has made great progress in recent years, the process of ILT has met obstacles. One is mask manufacturability, i.e., the lack of mask complexity control [18]: Most of the aforementioned approaches do not take this issue into account. Without the constraint of mask geometry, ILT is appreciated for its ability to freely manipulate pixels to achieve superior wafer image quality, but the resulting complex shapes render it impossible to manufacture the mask. The second obstacle is that most ILT algorithms focus on the mask performance only at nominal process conditions. However, the mechanical fluctuations of the imaging system [47] as well as algorithm variability [19] would lead to nonnegligible critical dimension (CD) variation and detection, which affect the mask pattern fidelity. The increased CD sensitivity to process variations needs to be handled to improve system performance. Some works have addressed this problem by considering the focus variation as a random variable, turning it into a stochastic optimization problem $[16,14,44]$. These works succeed in providing an extra margin for focus variation so that the system can adapt to different process conditions.

Motivated by the aforementioned obstacles and recent research on ILT, we propose a variational energy functional to tackle these obstacles and develop an effective computational algorithm for inverse photomask synthesis. Compared with the traditional formulation of inverse lithography, the merit of our proposed energy functional is that it takes into consideration the process variations and consists of penalty terms that can control the mask complexity. Thus the synthesized photomask is expected to be simple and more robust to variations of process conditions. In addition, we shall prove the existence of a minimizer of the energy functional. To minimize the proposed functional, we adopt a variable splitting technique and apply the alternating minimization method. Chambolle's fast duality projection algorithm [2] is used to solve the optimization problem.

This paper is organized as follows. In the next section, we briefly introduce the principle of an optical lithography imaging system. In section 3, we present the mathematical description of inverse lithography with focus variation. Sections 4 and 5 present the formulation of the

Copyright (c) by SIAM. Unauthorized reproduction of this article is prohibited. 


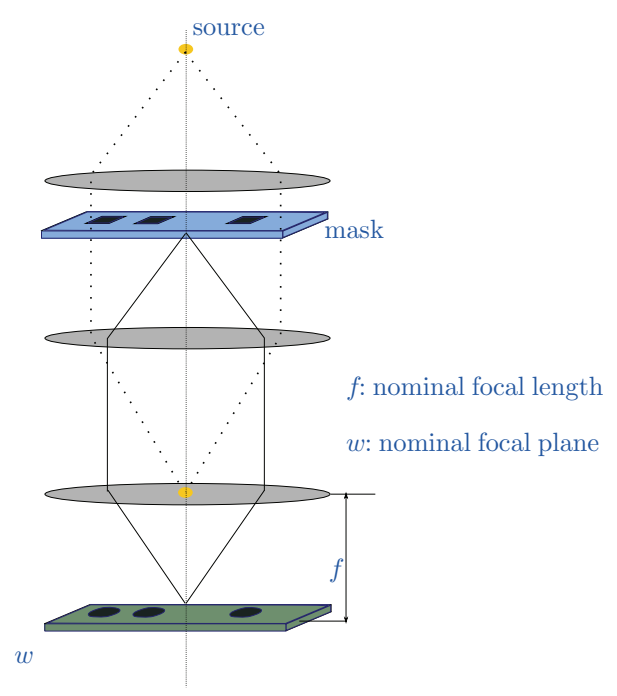

Figure 1. Imaging system of optical lithography.

proposed energy functional and the optimization procedure. Experimental results will be reported in section 6 . Section 7 discusses the extension of our proposed framework, which is followed by conclusions.

2. Principle of optical lithography. Optical lithography, as mentioned previously, uses light to print circuit patterns from mask to wafer. A typical lithography system includes four elements: an illuminator (a light source), a mask, an exposure system, and a silicon wafer [47], as shown in Figure 1. Light energy from the illuminator is delivered to the mask. Patterns on the mask are then replicated to the silicon wafer (at nominal focal plane) through the exposure system, which consists of a series of imaging lenses. The wafer is coated with a light-sensitive layer, on which regions with different levels of exposure are formed. This latent image on the wafer is then developed to have the low (or high) exposure regions removed for further processing. Assuming that the air, instead of the photo-resist wafer, occupies the space, the concept of the so-called aerial image is introduced to denote the virtual image formed in the air [25]. It serves as an approximator of the final image after development, which is represented by a gray-scale image in the following discussion due to its multiple intensity levels. In this paper, we also assume that the resist is ideal with infinite contrast so that the final image is binary.

3. Mathematical modeling of inverse lithography. The projection lithography imaging process can be described by the following forward model:

$$
I(x)=\Gamma\{U(x)\},
$$

where $x$ is the spatial image coordinates and $\Gamma\{\bullet\}$ is a function that maps the input intensity function $U(x)$ to the output intensity function $I(x)$. Let $I_{0}(x)$ be the desired image function (or target pattern). The objective of classical inverse lithography is to find an optimal input intensity function (an optimal mask), $\hat{U}(x)$, which minimizes the dissimilarity, $d$, between 


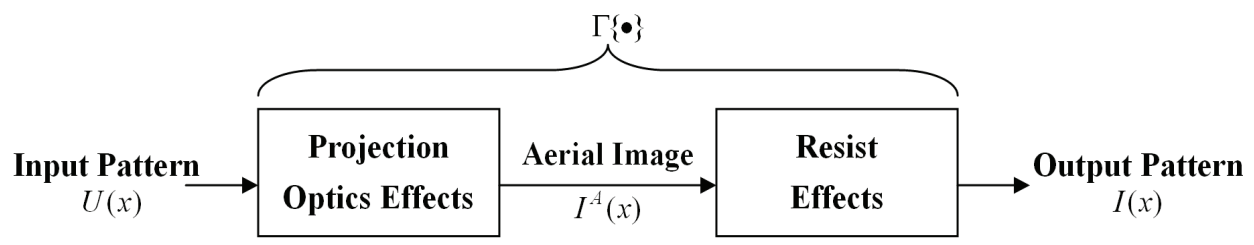

Figure 2. Forward model of the simplified lithography process.

$I_{0}(x)$ and $I(x)$ :

$$
\hat{U}(x)=\arg \min _{U(x)} d\left(I_{0}(x), \Gamma\{U(x)\}\right)
$$

where the $L^{2}$-norm is the widely used dissimilarity measure in the literature.

To compute $\hat{U}(x)$, we first mathematically express the image formation process (3.1). Figure 2 summarizes the forward model of the simplified lithography process, which consists of two functional blocks, namely, the projection optics effects (aerial image formation) and the resist effects. In this paper, we shall focus only on the coherent illumination and chromiumon-glass (CoG) binary mask, but our proposed method can be extended to apply to partially coherent imaging systems (see section 7.1 for more details) and phase-shifting masks.

Due to the intrinsic lowpass characteristic (diffraction) of the coherent imaging system, the aerial image intensity function $I^{A}(x)$ is typically a blurred version of $U(x)$. In other words, the projection optics effects can be regarded as a lowpass operation. Assume that there are no mechanical fluctuations for the imaging system (i.e., the real image plane always lies on the nominal focal plane). Then the aerial image can be approximated by

$$
I^{A}(x)=|(H * U)(x)|^{2} .
$$

The symbol " $*$ " denotes the convolution operator, and $H(x)$ is a (linear spatially invariant) point spread function that can be estimated by the inverse Fourier transform of the optical transfer function [14]:

$$
H(x)=\mathcal{F}^{-1}\{h(v)\}
$$

where

$$
h(v)= \begin{cases}1 & \text { if }\|v\|_{2} \leq N A / \lambda, \\ 0 & \text { else. }\end{cases}
$$

Here, $\|\bullet\|_{2}$ is the Euclidean norm, $v$ is the frequency variable, $N A$ is the numerical aperture of the imaging system, and $\lambda$ is the wavelength of the light source.

The resist effects are actually a series of chemical reactions (or the etching process) on the wafer, which can be simulated by various resist models such as a variable threshold resist model [37], a constant threshold resist model [13], and a logarithmic sigmoid resist model [35]. All these models involve a thresholding operation that removes the areas of aerial image intensity higher than a certain threshold. Here, we shall use the logarithmic sigmoid function, $\operatorname{sig}(\bullet)$, as the resist effects. Together with the projection optics effects, the image formation 
equation (3.1) can be written as

$$
I(x)=\operatorname{sig}\left(I^{A}(x)\right)=\frac{1}{1+\exp \left(-a\left(I^{A}(x)-t r\right)\right)},
$$

where $a$ controls the steepness of the sigmoid function, and $t r$ is the threshold. It is easy to see that the sigmoid function would resemble the Heaviside step function with threshold at $t r$ when $a$ is sufficiently large.

3.1. Inverse lithography with focus variation. In an ideal optical lithography system, the real image plane always lies on the nominal focal plane (see Figure 1). In practice, however, the real image plane no longer lies on the nominal focal plane but varies slightly around it, presumably due to mechanical fluctuations. As mentioned in section 1, such a focus error may significantly degrade the overall wafer image quality. Therefore, it is essential to control the lithography system so that it can adapt to focus variation.

Let $\beta$ be a random variable representing the distance between the real image plane and the nominal focal plane, and let $f$ be the nominal focal length between the lens and the nominal focal plane (see Figure 1). Then the real image plane will lie on the position in the range $f-\beta$ to $f+\beta$ measured from the lens. With focus variation $\beta$, the optical transfer function used in the lithography system can be modeled as $[16,14]$

$$
h_{\beta}(v)=h(v) \exp \left(-j \pi \beta\|v\|_{2}^{2}\right),
$$

which is equivalent to a shifting operation of the point spread function in the spatial domain. The defocus point spread function is then obtained by taking the inverse Fourier transform of (3.5), i.e., $H_{\beta}(x)=\mathcal{F}^{-1}\left\{h_{\beta}(v)\right\}$. As in (3.3) and (3.4), the aerial image intensity function and the output intensity function with focus variation are given by

$$
\begin{gathered}
I_{\beta}^{A}(x)=\left|\left(H_{\beta} * U\right)(x)\right|^{2}, \\
I_{\beta}(x)=\operatorname{sig}\left(I_{\beta}^{A}(x)\right)=\frac{1}{1+\exp \left(-a\left(I_{\beta}^{A}(x)-t r\right)\right)} .
\end{gathered}
$$

Given (3.6), (3.7), and a dissimilarity function $d$, our main objective is to compute the optimal mask $\hat{U}(x)$ by solving (3.2). Note that the minimization problem (3.2) involves deconvolution and is generally ill-posed. In this work, we propose solving the inverse lithography minimization problem with focus variation in a variational framework by incorporating the regularization functional into the formulation. In what follows, we shall simply neglect the argument $x$ whenever there is no ambiguity.

4. The proposed method. The optical proximity correction mask synthesis problem with focus variation can be formulated as follows: Given a desired image $I_{0}: \Omega \rightarrow \mathbb{R}$, where the image domain $\Omega$ is a bounded open subset of $\mathbb{R}^{2}$, the goal is to find a mask $U$ such that the output pattern $I_{\beta}$, which depends on $U$ and the defocus point spread function $H_{\beta}$, is approximately close to the given image $I_{0}$ by a certain suitable measure.

Copyright $\odot$ by SIAM. Unauthorized reproduction of this article is prohibited. 
In this paper, we propose defining the energy functional $P_{\beta}(U)$ as follows:

$$
\begin{aligned}
P_{\beta}(U)= & \frac{\lambda_{1}}{2} \int_{\Omega}\left(I_{\beta}-I_{0}\right)^{2} d x+\frac{\lambda_{2}}{2} \int_{\Omega}\left(U-I_{0}\right)^{2} d x+\lambda_{3} \int_{\Omega}|\nabla U| d x \\
& +\lambda_{4} \int_{\Omega}\left|\nabla I_{\beta}^{A}\right| d x+\frac{\lambda_{5}}{2} \int_{\Omega}\left(1-(2 U-1)^{2}\right) d x,
\end{aligned}
$$

where $\lambda_{i} \geq 0$ for all $i$ are constants, $\nabla$ is a gradient operator, and $I_{\beta}^{A}$ and $I_{\beta}$ are defined in (3.6) and (3.7), respectively. For the defocus point spread function $H_{\beta}$, we assume that $\int_{\Omega} H_{\beta} d x=1$. The interpretation of (4.1) is as follows: The first term is the cost function of the classical inverse lithography which requires that the output pattern approximates the target pattern and thus minimizes the pattern error; the second and third terms control the mask complexity globally and locally to ensure that the mask is easy to manufacture; the fourth term is the total variation of the aerial image which attempts to preserve the sharp edges of the aerial image and improves its overall contrast (or the tolerance to dose variation [47]); and the last term is a quadratic penalty that forces the mask to be closed to a binary pattern. We remark that while the second term can control the mask complexity globally, the value of $\lambda_{2}$ should be set sufficiently small. Otherwise, the optimal mask would resemble the target pattern and some useful assist features would be suppressed, leading to unsatisfactory pattern fidelity (see section 6.2 for more details).

We propose solving the inverse lithography problem with focus variation by minimizing the expectation of $P_{\beta}(U)$ :

$$
\mathbb{E}\left\{P_{\beta}(U)\right\}
$$

subject to the constraint

$$
0 \leq U \leq 1
$$

Note that we relax the original binary constraint $U \in\{0,1\}$ to $0 \leq U \leq 1$ such that the discrete optimization setting is transformed to the continuous optimization framework. In addition, the energy functional (4.1) is a highly nonlinear transcendental function, and thus its expectation is difficult to derive. Since the real image plane fluctuates around the nominal focal plane with focus variation $\beta$, it is reasonable to assume that the focus variation is zeromean Gaussian distributed with variance $\sigma^{2}$, i.e., $\beta \sim \mathcal{N}\left(0, \sigma^{2}\right)$. Given a collection of defocus samples $\left(\beta_{i}\right)_{i=1,2, \ldots, N}$, we shall approximate $(4.2)$ by the weighted sum of $P_{\beta_{i}}(U)$ using a technique similar to that in [14]:

$$
\mathbb{E}\left\{P_{\beta}(U)\right\} \approx \sum_{i=1}^{N} \eta_{i} P_{\beta_{i}}(U),
$$

where $\eta_{i}$ denotes the Gaussian density function of $\beta_{i}$. In this work, we solve the minimization problem (4.4) subject to the constraint (4.3).

Copyright (c) by SIAM. Unauthorized reproduction of this article is prohibited. 
4.1. Mathematical analysis. In this subsection, we shall prove the existence of a minimizer of (4.4). Since (4.4) is the weighted sum of (4.1), this is equivalent to proving the existence of a minimizer of the proposed energy functional (4.1) given a fixed defocus sample $\beta$. Under the assumption that $I_{0} \in B V(\Omega) \cap L^{\infty}(\Omega)$, where $B V(\Omega)$ is a space of functions of bounded variations, the energy $P_{\beta}(U)$ is well defined and finite for the set $\mathcal{S}_{U}=\{U \in B V(\Omega), 0 \leq U \leq 1\}$.

Theorem 4.1 (existence of a minimizer of $P_{\beta}$ ). Assume that $I_{\beta}^{A}, I_{0} \in B V(\Omega) \cap L^{\infty}(\Omega)$ and $\alpha_{1} \leq I_{\beta}^{A} \leq \alpha_{2}$. Then for fixed parameters $\lambda_{i}$ for all $i, \alpha_{1}, \alpha_{2}, \beta, a, t r$, there exists a minimizer of the energy $P_{\beta}$ in $\mathcal{S}_{U}$.

Proof. Let $U=0$; then $I_{\beta}^{A}=0$ and $I_{\beta}=1 /(1+\exp (a \cdot t r)) \in(0,1)$, and thus $\left.P_{\beta}\right|_{U=0}=$ $\frac{\lambda_{1}}{2} \int_{\Omega}\left(I_{\beta}-I_{0}\right)^{2} d x+\frac{\lambda_{2}}{2} \int_{\Omega} I_{0}^{2} d x<+\infty$. Since $P_{\beta} \geq 0$, we deduce that the infimum of the energy $P_{\beta}$ must be finite. Let $\left\{U^{n}\right\} \subseteq \mathcal{S}_{U}$ be a minimizing sequence for the energy $P_{\beta}$, i.e., $P_{\beta}\left(U^{n}\right) \rightarrow \inf P_{\beta}(U)$ as $n \rightarrow \infty$. Then there exists a constant $M>0$ such that

$$
\begin{aligned}
P_{\beta}\left(U^{n}\right)= & \frac{\lambda_{1}}{2} \int_{\Omega}\left(I_{\beta}^{n}-I_{0}\right)^{2} d x+\frac{\lambda_{2}}{2} \int_{\Omega}\left(U^{n}-I_{0}\right)^{2} d x+\lambda_{3} \int_{\Omega}\left|\nabla U^{n}\right| d x \\
& +\lambda_{4} \int_{\Omega}\left|\nabla\left(I_{\beta}^{A}\right)^{n}\right| d x+\frac{\lambda_{5}}{2} \int_{\Omega}\left(1-\left(2 U^{n}-1\right)^{2}\right) d x \leq M,
\end{aligned}
$$

where $\left(I_{\beta}^{A}\right)^{n}=\left|H_{\beta} * U^{n}\right|^{2}$ and $I_{\beta}^{n}=\operatorname{sig}\left(\left(I_{\beta}^{A}\right)^{n}\right)$. This implies that each term of $P_{\beta}\left(U^{n}\right)$ is bounded by $M$.

Since $0 \leq U^{n} \leq 1,\left\|U^{n}\right\|_{L^{1}(\Omega)}=\int_{\Omega} U^{n} d x \leq|\Omega|$. Together with $\int_{\Omega}\left|\nabla U^{n}\right| d x \leq M$, we see that $\left\{U^{n}\right\}$ is uniformly bounded in $B V(\Omega)$. Since every uniformly bounded sequence in $B V(\Omega)$ is relatively compact in $L^{1}(\Omega)$, there exist a subsequence (also denoted by) $\left\{U^{n}\right\}$ and a function $U^{*} \in B V(\Omega)$ such that

$$
\begin{aligned}
U^{n} \rightarrow U^{*} & \text { strongly in } L^{1}(\Omega), \\
U^{n} \rightarrow U^{*} & \text { a.e. } x \in \Omega, \\
\nabla U^{n} \rightarrow \nabla U^{*} & \text { in the sense of measure. }
\end{aligned}
$$

By the lower semicontinuity of total variation and the $L^{2}$-norm and Fatou's lemma, we have

$$
\begin{gathered}
\int_{\Omega}\left|\nabla U^{*}\right| d x \leq \liminf _{n \rightarrow \infty} \int_{\Omega}\left|\nabla U^{n}\right| d x, \\
\int_{\Omega}\left(U^{*}-I_{0}\right)^{2} d x \leq \liminf _{n \rightarrow \infty} \int_{\Omega}\left(U^{n}-I_{0}\right)^{2} d x, \\
\int_{\Omega}\left(1-\left(2 U^{*}-1\right)^{2}\right) d x \leq \liminf _{n \rightarrow \infty} \int_{\Omega}\left(1-\left(2 U^{n}-1\right)^{2}\right) d x .
\end{gathered}
$$

Since $\int_{\Omega}\left|\nabla\left(I_{\beta}^{A}\right)^{n}\right| d x \leq M$ and $\alpha_{1} \leq\left(I_{\beta}^{A}\right)^{n} \leq \alpha_{2}$, we have that the total variation and the $L^{1}$-norm of $\left(I_{\beta}^{A}\right)^{n}$ are uniformly bounded. Then $\left\{\left(I_{\beta}^{A}\right)^{n}\right\}$ is uniformly bounded in $B V(\Omega)$, and there exist a subsequence (also denoted by) $\left\{\left(I_{\beta}^{A}\right)^{n}\right\}$ and a function $\left(I_{\beta}^{A}\right)^{*} \in B V(\Omega)$, 
$\alpha_{1} \leq\left(I_{\beta}^{A}\right)^{*} \leq \alpha_{2}$, such that

$$
\begin{gathered}
\left(I_{\beta}^{A}\right)^{n} \rightarrow\left(I_{\beta}^{A}\right)^{*} \quad \text { strongly in } L^{1}(\Omega), \\
\left(I_{\beta}^{A}\right)^{n} \rightarrow\left(I_{\beta}^{A}\right)^{*} \quad \text { a.e. } x \in \Omega, \\
\nabla\left(I_{\beta}^{A}\right)^{n} \rightarrow \nabla\left(I_{\beta}^{A}\right)^{*} \quad \text { in the sense of measure. }
\end{gathered}
$$

The lower semicontinuity of total variation gives

$$
\int_{\Omega}\left|\nabla\left(I_{\beta}^{A}\right)^{*}\right| d x \leq \liminf _{n \rightarrow \infty} \int_{\Omega}\left|\nabla\left(I_{\beta}^{A}\right)^{n}\right| d x
$$

Since $I_{\beta}^{n}=\operatorname{sig}\left(\left(I_{\beta}^{A}\right)^{n}\right) \in(0,1)$ and $\left(I_{\beta}^{A}\right)^{n} \rightarrow\left(I_{\beta}^{A}\right)^{*}$ a.e. $x \in \Omega$, from the boundedness of the sequence $\left\{I_{\beta}^{n}\right\}$, we can extract a subsequence (also denoted by) $\left\{I_{\beta}^{n}\right\}$ and a function $I_{\beta}^{*}$ such that

$$
I_{\beta}^{n}=\operatorname{sig}\left(\left(I_{\beta}^{A}\right)^{n}\right) \rightarrow \operatorname{sig}\left(\left(I_{\beta}^{A}\right)^{*}\right)=I_{\beta}^{*} \quad \text { uniformly. }
$$

By the lower semicontinuity of the $L^{2}$-norm, we have

$$
\int_{\Omega}\left(I_{\beta}^{*}-I_{0}\right)^{2} d x \leq \liminf _{n \rightarrow \infty} \int_{\Omega}\left(I_{\beta}^{n}-I_{0}\right)^{2} d x .
$$

Combining (4.5)-(4.9), we have established that

$$
P_{\beta}\left(U^{*}\right) \leq \liminf _{n \rightarrow \infty} P_{\beta}\left(U^{n}\right)=\inf P_{\beta}(U),
$$

and hence $U^{*}$ must be a minimizer.

5. The optimization procedure. Minimization of (4.4) with the constraint (4.3) forms a class of constrained nonlinear optimization problems whose solutions are unknown. In order to effectively minimize the energy functional subject to the constraint, we adopt an alternating minimization procedure and make use of Chambolle's fast duality projection algorithm [2]. The data fidelity and the total variation terms in the functional can be decoupled by introducing auxiliary variables $V$ and $J_{\beta}^{A}=\left(J_{\beta_{i}}^{A}\right)_{i=1,2, \ldots, N}$. Given a set of defocus samples $\left(\beta_{i}\right)_{i=1,2, \ldots, N}$, we shall approximate $(4.4)$ by replacing $U$ and $I_{\beta}^{A}=\left(I_{\beta_{i}}^{A}\right)_{i=1,2, \ldots, N}$ with $V$ and $J_{\beta}^{A}$, respectively, in the total variation terms of (4.1) and adding convex terms that force $I_{\beta}^{A}$ and $J_{\beta}^{A}$, and $U$ and $V$, to be sufficiently close:

$$
\begin{aligned}
Q_{\beta}\left(U, V, J_{\beta}^{A}\right)= & \sum_{i=1}^{N} \eta_{i}\left(\frac{\lambda_{1}}{2} \int_{\Omega}\left(I_{\beta_{i}}-I_{0}\right)^{2} d x+\frac{\lambda_{2}}{2} \int_{\Omega}\left(U-I_{0}\right)^{2} d x+\lambda_{3} \int_{\Omega}|\nabla V| d x\right. \\
& +\frac{1}{2 \theta_{1}} \int_{\Omega}(V-U)^{2} d x+\lambda_{4} \int_{\Omega}\left|\nabla J_{\beta_{i}}^{A}\right| d x+\frac{1}{2 \theta_{2}} \int_{\Omega}\left(J_{\beta_{i}}^{A}-I_{\beta_{i}}^{A}\right)^{2} d x \\
& \left.+\frac{\lambda_{5}}{2} \int_{\Omega}\left(1-(2 U-1)^{2}\right) d x\right),
\end{aligned}
$$

where $\theta_{1}$ and $\theta_{2}$ are small positive constants. We remark that when $\theta_{1}$ and $\theta_{2}$ are sufficiently small, we force $V$ close to $U$, and $J_{\beta}^{A}$ close to $I_{\beta}^{A}$. Therefore, minimizing (5.1) can be regarded as a minimization of (4.4) when $\theta_{1}$ and $\theta_{2}$ are sufficiently small with respect to the $L^{2}$-norm. In the following, we shall present the alternating minimization procedure (sections 5.1-5.3) and the overall implementation of the mask synthesis algorithm (section 5.4).

Copyright ( $\odot$ by SIAM. Unauthorized reproduction of this article is prohibited. 
5.1. Solving for auxiliary variable $\boldsymbol{V}$. Fixing $U$ and $J_{\beta}^{A}$, we compute $V$ by minimizing

$$
\int_{\Omega}|\nabla V| d x+\frac{1}{2 \delta_{1}} \int_{\Omega}(V-U)^{2} d x
$$

where $\delta_{1}=\lambda_{3} \cdot \theta_{1}$. This is the well-known Rudin-Osher-Fatemi model [41], which can be solved by many different methods such as Chambolle's fast duality projection algorithm $[2,7]$, semismooth Newton's method [27], and the multilevel optimization method [5]. In this paper, we employ the fast duality projection algorithm of Chambolle due to its efficiency, effectiveness, ease of implementation [20], and high numerical stability [26]. Then the solution is given by

$$
\hat{V}=U-\delta_{1} \operatorname{div} p_{1},
$$

where div is a divergence operator and $p_{1}$ can be computed using a fixed point algorithm:

$$
p_{1}^{n+1}=\frac{p_{1}^{n}+\tau_{1} \nabla\left(\operatorname{div} p_{1}^{n}-U / \delta_{1}\right)}{1+\tau_{1}\left|\nabla\left(\operatorname{div} p_{1}^{n}-U / \delta_{1}\right)\right|}, \quad n=0,1, \ldots
$$

with initial value $p_{1}^{0}=0$. Since the algorithm is guaranteed to converge for $0<\tau_{1} \leq 1 / 8$ [2], we simply choose $\tau_{1}=0.025$. In addition, we choose $\theta_{1}=0.01$ in all the following experiments. ${ }^{1}$

5.2. Solving for auxiliary variable $J_{\beta}^{A}$. Fixing $U$ and $V$, we compute $J_{\beta_{i}}^{A}$ by minimizing

$$
\int_{\Omega}\left|\nabla J_{\beta_{i}}^{A}\right| d x+\frac{1}{2 \delta_{2}} \int_{\Omega}\left(J_{\beta_{i}}^{A}-I_{\beta_{i}}^{A}\right)^{2} d x
$$

where $\delta_{2}=\lambda_{4} \cdot \theta_{2}$. Using the same technique as in section 5.1, the solution is given by

$$
\hat{J}_{\beta_{i}}^{A}=I_{\beta_{i}}^{A}-\delta_{2} \operatorname{div} p_{2, i},
$$

where $p_{2, i}$ can be determined using a fixed point algorithm, provided that $\delta_{2}$ and $\tau_{2}$ are fixed. Following the parameter specification as in section 5.1, we simply choose $\theta_{2}=\theta_{1}$ and $\tau_{2}=\tau_{1}$ in all our experiments.

5.3. Solving for mask $U$. Fixing $V$ and $J_{\beta}^{A}$, we solve $U$ by minimizing

$$
\begin{aligned}
\widetilde{Q}_{\beta}(U)= & \sum_{i=1}^{N} \eta_{i}\left(\frac{\lambda_{1}}{2} \int_{\Omega}\left(I_{\beta_{i}}-I_{0}\right)^{2} d x+\frac{\lambda_{2}}{2} \int_{\Omega}\left(U-I_{0}\right)^{2} d x+\frac{1}{2 \theta_{1}} \int_{\Omega}(V-U)^{2} d x\right. \\
& \left.+\frac{1}{2 \theta_{2}} \int_{\Omega}\left(J_{\beta_{i}}^{A}-I_{\beta_{i}}^{A}\right)^{2} d x+\frac{\lambda_{5}}{2} \int_{\Omega}\left(1-(2 U-1)^{2}\right) d x\right)
\end{aligned}
$$

subject to

$$
0 \leq U \leq 1
$$

\footnotetext{
${ }^{1}$ We also found that $0<\theta_{1}<0.5$ achieved satisfactory optimal masks and output patterns. As $\theta_{1}=0.01$ usually gave the best results, we simply report the $\theta_{1}=0.01$ case in our experiments.
}

Copyright (c) by SIAM. Unauthorized reproduction of this article is prohibited. 
If $\tilde{U}$ is a minimizer of $\widetilde{Q}_{\beta}$, the gradient of $\widetilde{Q}_{\beta}$ with respect to $\tilde{U}$ must vanish. Then we have

$$
\frac{\partial \widetilde{Q}_{\beta}}{\partial \tilde{U}}=\sum_{i=1}^{N} \eta_{i} g_{\beta_{i}}(\tilde{U})=0
$$

where

$$
\begin{aligned}
g_{\beta_{i}}(\tilde{U})= & H_{\beta_{i}} *\left(\left(H_{\beta_{i}}^{\dagger} * \tilde{U}\right) \bullet \Im_{i}^{1}\right)+H_{\beta_{i}}^{\dagger} *\left(\left(H_{\beta_{i}} * \tilde{U}\right) \bullet \Im_{i}^{1}\right) \\
& +\left(\frac{1}{\theta_{1}}(\tilde{U}-V)+\lambda_{2}\left(\tilde{U}-I_{0}\right)+\lambda_{5}(2-4 \tilde{U})\right), \\
\Im_{i}^{1}= & a \lambda_{1} I_{\beta_{i}} \bullet\left(1-I_{\beta_{i}}\right) \bullet\left(I_{\beta_{i}}-I_{0}\right)+\frac{1}{\theta_{2}}\left(I_{\beta_{i}}^{A}-J_{\beta_{i}}^{A}\right) .
\end{aligned}
$$

For the derivation of (5.6), we refer the reader to Appendix A. In (5.7), the symbol "•" denotes pointwise multiplication, and $H_{\beta_{i}}^{\dagger}$ is the complex conjugate of $H_{\beta_{i}}$. Note that (5.6) is a transcendental equation and can be solved numerically. We shall use the Newton-Raphson algorithm for computing $\tilde{U}$.

Define the middle part of (5.6) as a function of $\tilde{U}, f_{\beta}(\tilde{U})$, i.e., $f_{\beta}(\tilde{U})=\sum_{i=1}^{N} \eta_{i} g_{\beta_{i}}(\tilde{U})$. Differentiating $f_{\beta}(\tilde{U})$ with respect to $\tilde{U}$ gives

$$
f_{\beta}^{\prime}(\tilde{U})=\sum_{i=1}^{N} \eta_{i} g_{\beta_{i}}^{\prime}(\tilde{U})
$$

where

$$
\begin{aligned}
g_{\beta_{i}}^{\prime}(\tilde{U})= & 2\left(H_{\beta_{i}} \bullet H_{\beta_{i}}^{\dagger}\right) *\left(\Im_{i}^{1}+\Im_{i}^{2} \bullet I_{\beta_{i}}^{A}\right)+H_{\beta_{i}}^{2} *\left(\left(H_{\beta_{i}}^{\dagger} * \tilde{U}\right)^{2} \bullet \Im_{i}^{2}\right) \\
& +\left(H_{\beta_{i}}^{\dagger}\right)^{2} *\left(\left(H_{\beta_{i}} * \tilde{U}\right)^{2} \bullet \Im_{i}^{2}\right)+\left(\frac{1}{\theta_{1}}+\lambda_{2}-4 \lambda_{5}\right), \\
\Im_{i}^{2}= & a^{2} \lambda_{1} I_{\beta_{i}} \bullet\left(1-I_{\beta_{i}}\right) \bullet\left(I_{\beta_{i}} \bullet\left(1-I_{\beta_{i}}\right)+\left(I_{\beta_{i}}-I_{0}\right) \bullet\left(1-2 I_{\beta_{i}}\right)\right)+\frac{1}{\theta_{2}} .
\end{aligned}
$$

The derivation of (5.8) can be obtained using similar techniques as in Appendix A. Then the minimizer $\tilde{U}$ is obtained by

$$
\tilde{U}^{n+1}=\tilde{U}^{n}-\frac{f_{\beta}\left(\tilde{U}^{n}\right)}{f_{\beta}^{\prime}\left(\tilde{U}^{n}\right)}, \quad n=0,1, \ldots
$$

Note that, in each iteration of the Newton-Raphson algorithm, $\tilde{U}$ is updated after computing the gradients for all the defocus samples $\left(\beta_{i}\right)_{i=1,2, \ldots, N}$, which is computationally expensive. Here we shall adopt a more efficient way to compute the minimizer. Specifically, instead of considering all the defocus samples for each iteration step, we simply update $\tilde{U}$ using a single defocus sample $\beta_{i}$ which is randomly generated from $\mathcal{N}\left(0, \sigma^{2}\right)$. Then the minimizer is

$$
\tilde{U}^{n+1}=\tilde{U}^{n}-\frac{g_{\beta_{i}}\left(\tilde{U}^{n}\right)}{g_{\beta_{i}}^{\prime}\left(\tilde{U}^{n}\right)}, \quad n=0,1, \ldots
$$

Copyright (c) by SIAM. Unauthorized reproduction of this article is prohibited. 
By using a different defocus sample in each iteration step, the estimated mask is adjusted continuously to adapt to different defocus conditions, and, thus, the lithography system is more robust in a certain range (depends on the variance of $\beta_{i}$ ) of focus errors. We also remark that the ratio $g_{\beta_{i}}\left(\tilde{U}^{n}\right) / g_{\beta_{i}}^{\prime}\left(\tilde{U}^{n}\right)$ is independent of the Gaussian density function $\eta_{i}$. Once the mask $\tilde{U}$ is computed from (5.9), the solution $\hat{U}$ is constructed by projecting $\tilde{U}$ onto $[0,1]$ such that the constraint (5.5) is satisfied:

$$
\hat{U}=\min \{\max \{\tilde{U}, 0\}, 1\} .
$$

5.4. Algorithm. The overall implementation of the mask synthesis algorithm is summarized as follows:

- Step 1: Initialize the mask $U$.

- Step 2: Update the auxiliary variables $\hat{V}$ and $\hat{J}_{\beta_{i}}^{A}$ using (5.2) and (5.3).

- Step 3: Update $\hat{U}$ using (5.10).

Repeat Step 2 and Step 3 until termination. The iteration stops if one of the following criteria is satisfied:

$$
\int_{\Omega}\left(\hat{U}^{\text {new }}-\hat{U}^{\text {old }}\right)^{2} d x<\varepsilon \quad \text { or } \quad \text { Ite }<K,
$$

where $\varepsilon$ is a small positive constant, Ite is the number of iterations of the algorithm, and $K$ is a positive integer.

- Step 4: Given $\hat{U}(x) \in[0,1], x \in \Omega$, obtained from Step 3, the optimal binary mask $\hat{U}^{o p t}(x) \in\{0,1\}$ is constructed by

$$
\hat{U}^{o p t}(x)= \begin{cases}1 & \text { if } \hat{U}(x)>t m, \\ 0 & \text { else }\end{cases}
$$

where $t m=0.5$.

Note that in Step 4 of the above mask synthesis algorithm, $t m=0.5$ corresponds to the value that maximizes the term $\int_{\Omega}\left(1-(2 U-1)^{2}\right) d x$ in our proposed functional (4.1). Thus, this value can be used as a natural threshold to binarize the mask $\hat{U} \in[0,1]$. We also remark that in Step $2, p_{1}$ and $p_{2, i}$ are computed in order to update the variables $\hat{V}$ and $\hat{J}_{\beta_{i}}^{A}$. Here, we iterate the fixed point algorithm only one time with the initial values $p_{1}^{0}$ and $p_{2, i}^{0}$ equal to the last loop of the alternating minimization to estimate $p_{1}$ and $p_{2, i}$. This setting makes use of the information of $p_{1}$ and $p_{2, i}$ obtained in the last loop in the minimization process and is presumably more efficient and effective. Similarly, we shall iterate the Newton-Raphson algorithm only one time (given the updated auxiliary variables) to obtain $\tilde{U}$ (see (5.9)). Moreover, instead of employing the steepest descent algorithm, as used in the traditional inverse lithography problem, to solve for $\tilde{U}$, we adopt the Newton-Raphson algorithm to compute $\tilde{U}$, which avoids choosing a constant step size that may yield poor results.

6. Experiments. We apply our proposed methodology to design some CoG binary mask patterns. Unless otherwise specified, we fix the parameters in all our experiments as follows: $\lambda=193 \mathrm{~nm}, N A=0.85$, resolution $=10 \mathrm{~nm} /$ pixel, $a=90, t r=0.3$, and the size of $H_{\beta}$ is $51 \times 51$. We simply choose these values as they are often adopted in the literature; see, for 
instance, $[14,16,33,43]$. For the regularization parameters, we note that $\lambda_{1}$ and $\lambda_{4}$ are the parameters corresponding to the pattern fidelity and aerial image contrast penalty terms which would affect the accuracy of the output pattern. Therefore, they should be set sufficiently large compared to other regularization parameters. On the other hand, $\lambda_{3}$ and $\lambda_{5}$ are the parameters of the local complexity and quadratic penalty terms which mainly influence the structure of the optimal masks (and also affect the pattern fidelity indirectly). Thus, they are presumably less important than $\lambda_{1}$ and $\lambda_{4}$. For the parameter of global complexity penalty term $\lambda_{2}$, as we mentioned earlier in section 4 , it should be set sufficiently small; otherwise, the optimal mask would resemble the target pattern leading to unsatisfactory pattern fidelity. Nevertheless, a well-chosen $\lambda_{2}$ can make the optimal mask less complex. Generally speaking, we suggest that the regularization parameters should be set according to $\lambda_{1}>\lambda_{4} \geq \lambda_{3} \approx \lambda_{5}>\lambda_{2}$. In this paper, we simply choose $\lambda_{1}=10.0, \lambda_{3}=1.0, \lambda_{4}=3.0, \lambda_{5}=1.0$, whereas the parameter $0 \leq \lambda_{2}<1$ is required to be tuned for each case. The choice of these parameters seems to give the best results in our experiments. ${ }^{2}$ The parameters of the stopping criteria of the mask synthesis algorithm, $\varepsilon$ and $K$, can be set arbitrarily by the user. Here, we shall set $\varepsilon=0.005$ and $K=300$. In addition, the defocus sample is assumed to be zero-mean Gaussian distributed with variance $\sigma^{2}$. In practice, the standard deviation would not be too large, and setting $\sigma=150 \mathrm{~nm}$ would be good enough to capture the most probable range of fluctuations of the real image plane. In the proposed algorithm, only the mask needs to be initialized. A trivial guess for the initial mask is the target pattern itself (i.e., $U^{\text {init }}=I_{0}$ ). However, the major disadvantage of such an initialization is that the algorithm tends to search for a local minimum around the target pattern, which may lead to unsatisfactory fidelity [4]. Here, the initial mask is generated based on the uniform distribution to ensure that the constraint (4.3) is satisfied. For simplicity, we shall refer to it as $U^{i n i t}=U D$ hereafter. To quantify the effectiveness of our proposed method, we use a pattern error, $P_{e}$, defined as the number of pixel differences between the target pattern and the output pattern:

$$
P_{e}=\left\|I_{0}-I_{\beta}\right\|_{F}^{2},
$$

where $\|\bullet\|_{F}$ is the Frobenius norm.

All experiments have been implemented on a Pentium IV PC (2 GHz) in a MATLAB environment. Essentially, the computational cost of the proposed mask synthesis algorithm mainly depends on solving $\tilde{U}$ in (5.9) using the Newton-Raphson algorithm. For each image shown below, the computation time to synthesize the optimal mask is about 60-300 seconds. We emphasize that our main goal is to propose a robust and effective computational algorithm to synthesize a simple mask which gives a small pattern error. Therefore the computational cost is not a major issue in this paper. Moreover, in all the following experiments (Figures 3-6 and Figures 9-11), the target patterns are chosen such that their critical dimensions lie in the range $[80 \mathrm{~nm}, 130 \mathrm{~nm}]$ and are below the Rayleigh criterion $R=0.61 \lambda / N A \approx 140 \mathrm{~nm}$.

We start by comparing the results of the proposed method at the best focus and under defocus when the inputs are target pattern and optimal mask, respectively. Then we compare our methodology with some existing approaches. We remark that, for all binary images

\footnotetext{
${ }^{2}$ We also found that $\lambda_{1} \in[5,12], \lambda_{2} \in[0,0.5], \lambda_{3} \in[0.5,1.5], \lambda_{4} \in[1.5,4]$, and $\lambda_{5} \in[0.5,1.5]$ gave similar and satisfactory results in all our experiments.
}

Copyright (c) by SIAM. Unauthorized reproduction of this article is prohibited. 


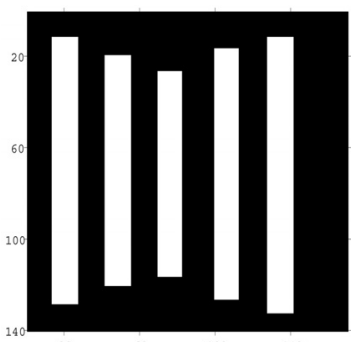

(a)

(d)

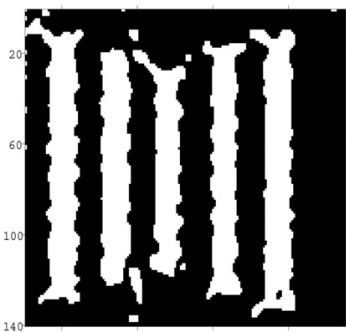

(b)
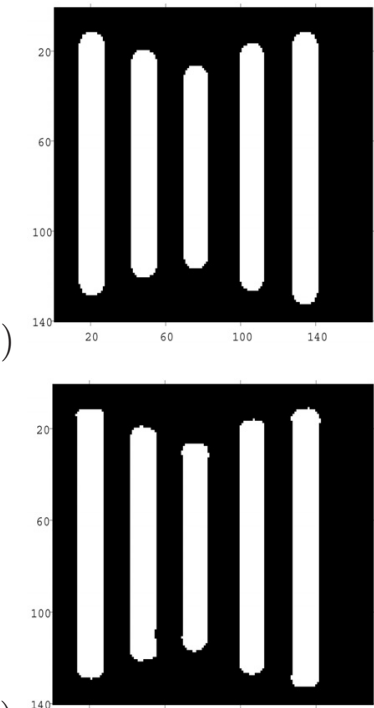

(e)

Figure 3. (a) Target pattern of size $170 \times 140$ pixels. (b)-(c) Output patterns at the best focus and under defocus 300nm with target pattern as input. (d) Optimal mask generated by our method. (e)-(f) Output patterns at the best focus and under defocus 300nm with optimal mask as input.

shown below, the black regions represent the unexposed region on the wafer, whereas the white regions correspond to the printed patterns on the wafer.

6.1. Effectiveness of our methodology. We first illustrate the significance of mask optimization by comparing the output patterns when the inputs are target pattern and optimal mask synthesized by our method, respectively. Figures $3-6$ show the comparative results at the best focus $\beta=0 \mathrm{~nm}$ and under defocus $\beta=300 \mathrm{~nm}$. Here, we shall choose $\lambda_{2}=0$ in these four experiments, and therefore, we control the mask complexity only locally (note that in section 6.2 , we shall discuss the impact of a global complexity penalty on the structure of the optimal mask and the pattern fidelity). In Figures 3-6, the top row displays the results using the target pattern as input, whereas the bottom row is the results using the synthesized mask as input.

Figure 3(a) shows the target pattern consisting of five vertical rectangular bars. Figures 3(b)-3(c) display the output patterns at the best focus and under defocus, respectively, with target pattern as input. Compared with the desired target pattern, we observe that the errors for Figures 3(b)-3(c) are visually noticeable, especially for the regions located at the end of each rectangular bar. Figure 3(d) shows the optimal mask generated by our proposed method, and Figures 3(e)-3(f) are the corresponding output patterns. As can be seen in Figure 3(d), some assist features near the vertical bars are automatically generated in the minimization process, which would help to improve the overall pattern fidelity. In addition, the cornerrounding effect at the end of each bar shown in Figures $3(\mathrm{e})-3(\mathrm{f})$ is reduced compared to Figures 3(b)-3(c), and the optimal mask gives the satisfactory contours in the output patterns. To quantitatively assess the pattern fidelity, we shall compare their pattern errors (and also their corresponding percentage errors) at the best focus and under defocus conditions, as

Copyright (c) by SIAM. Unauthorized reproduction of this article is prohibited. 
Table 1

Pattern errors and the corresponding percentage errors of our method at the best focus and under defocus $300 \mathrm{~nm}$ when the inputs are target pattern and optimal mask.

\begin{tabular}{c||cc|cc}
\hline \hline \multirow{2}{*}{ Figure } & \multicolumn{2}{c|}{ Target pattern } & \multicolumn{2}{c}{ Optimal mask } \\
\cline { 2 - 5 } & $\beta=0$ & $\beta=300$ & $\beta=0$ & $\beta=300$ \\
\hline 3 & 158 & 260 & 82 & 163 \\
& $(0.66 \%)$ & $(1.09 \%)$ & $(0.34 \%)$ & $(0.68 \%)$ \\
\hline 4 & 679 & 781 & 47 & 256 \\
& $(2.85 \%)$ & $(3.28 \%)$ & $(0.20 \%)$ & $(1.08 \%)$ \\
\hline 5 & 857 & 1116 & 228 & 432 \\
& $(2.28 \%)$ & $(2.97 \%)$ & $(0.61 \%)$ & $(1.15 \%)$ \\
\hline 6 & 592 & 1311 & 273 & 636 \\
& $(1.48 \%)$ & $(3.28 \%)$ & $(0.68 \%)$ & $(1.59 \%)$ \\
\hline \hline
\end{tabular}

(a)

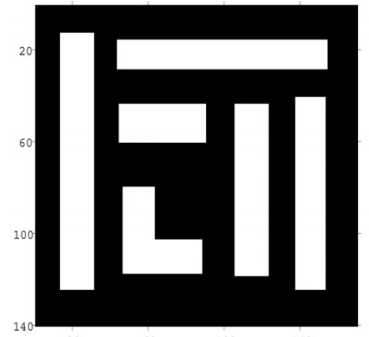

(b)

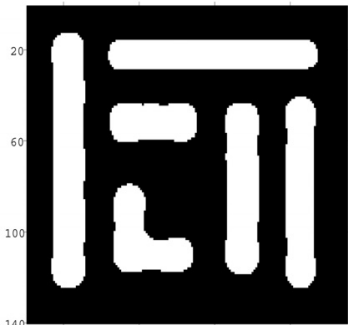

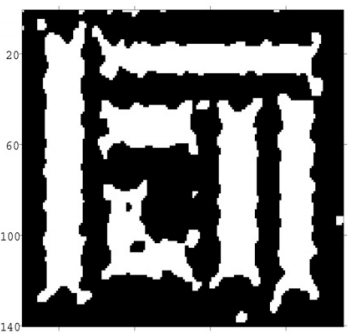

(d)

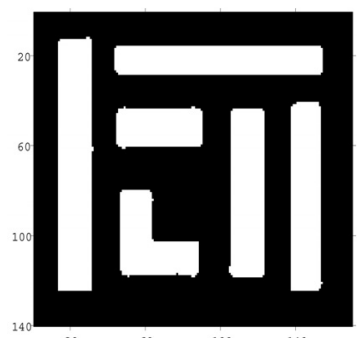

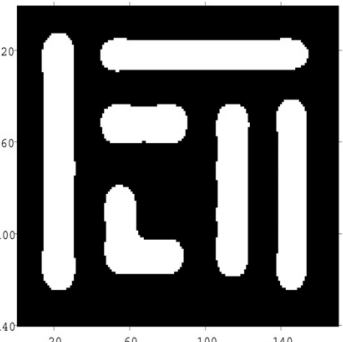

(c)

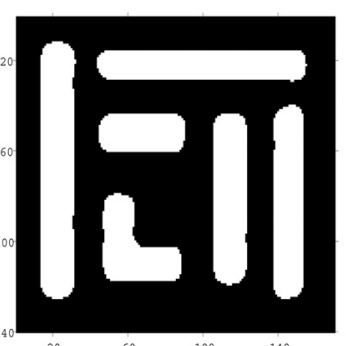

Figure 4. (a) Target pattern of size $170 \times 140$ pixels. (b)-(c) Output patterns at the best focus and under defocus $300 \mathrm{~nm}$ with target pattern as input. (d) Optimal mask generated by our method. (e)-(f) Output patterns at the best focus and under defocus $300 \mathrm{~nm}$ with optimal mask as input.

reported in Table 1 . With the target pattern as input, the pattern errors (and percentage errors) for Figures $3(\mathrm{~b})-3(\mathrm{c})$ are $P_{e}=158(0.66 \%)$ and $P_{e}=260(1.09 \%)$, respectively, while $P_{e}=82(0.34 \%)$ and $P_{e}=163(0.68 \%)$ for Figures $3(\mathrm{e})-3(\mathrm{f})$ when the input is the optimal mask. This reveals that the optimal mask generated by our method gives a more accurate pattern fidelity compared with using the target pattern as input. Moreover, our approach is also robust to focus variation in the sense that the proposed algorithm gives a promising output pattern even when a $300 \mathrm{~nm}$ focus error is introduced.

Figure 4 shows another example where the target pattern is composed of vertical and horizontal rectangular bars. As far as the pattern fidelity is concerned, the pattern errors (and percentage errors) for Figures $4(\mathrm{~b})-4(\mathrm{c})$ are about 14 times $\left(P_{e}=679(2.85 \%)\right.$ versus $\left.P_{e}=47(0.20 \%)\right)$ and 3 times $\left(P_{e}=781(3.28 \%)\right.$ versus $\left.P_{e}=256(1.08 \%)\right)$ higher than those 
(a)

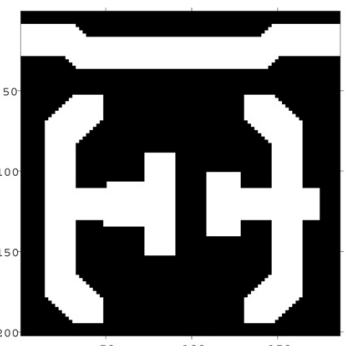

(d)

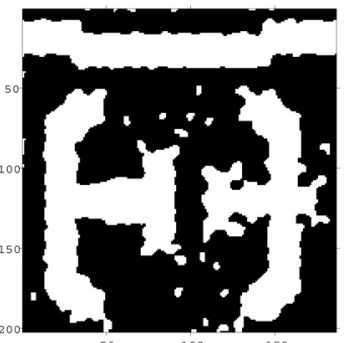

(b)
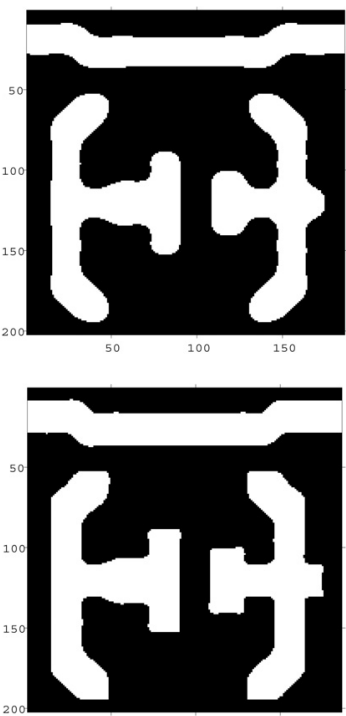

(c)

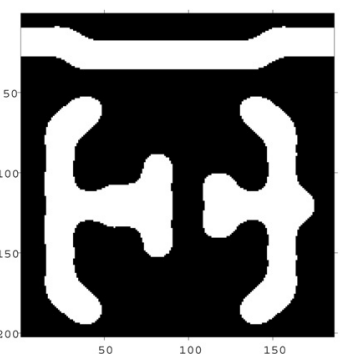

(e)

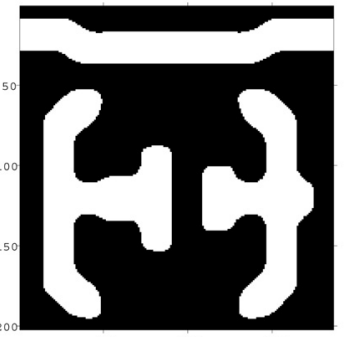

(f)

Figure 5. (a) Target pattern of size $186 \times 202$ pixels. (b)-(c) Output patterns at the best focus and under defocus 300nm with target pattern as input. (d) Optimal mask generated by our method. (e)-(f) Output patterns at the best focus and under defocus 300nm with optimal mask as input.

(a)

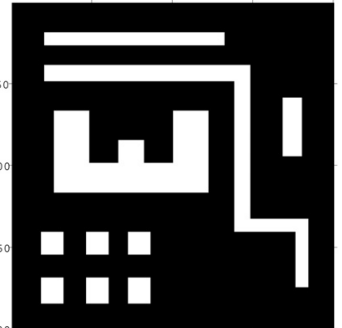

$1 \mathrm{O}(\mathrm{b})$

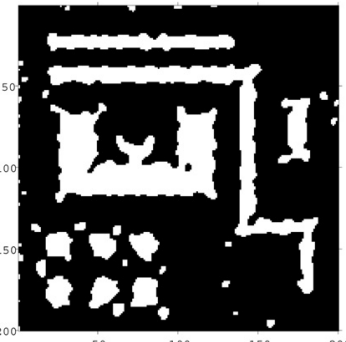

(d)

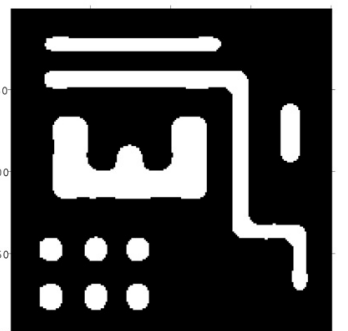

$200(\mathrm{c})$

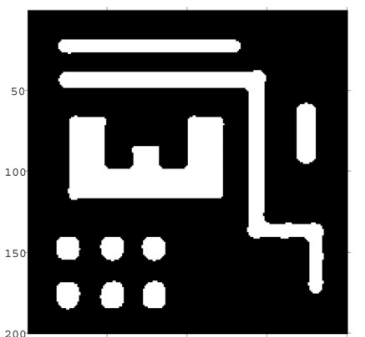

(e)
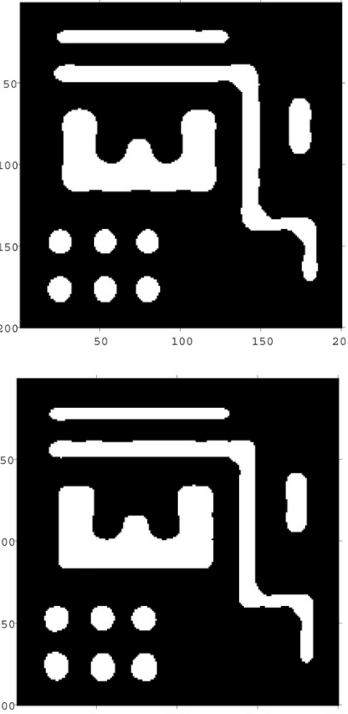

Figure 6. (a) Target pattern of size $200 \times 200$ pixels. (b)-(c) Output patterns at the best focus and under defocus $300 \mathrm{~nm}$ with target pattern as input. (d) Optimal mask generated by our method. (e)-(f) Output patterns at the best focus and under defocus 300nm with optimal mask as input.

for Figures 4(e)-4(f), respectively. This clearly indicates the importance of mask optimization.

The next two examples, Figures 5-6, show the more complicated structures in which the target patterns are composed of various polygons. When the input is target pattern, 
(a)

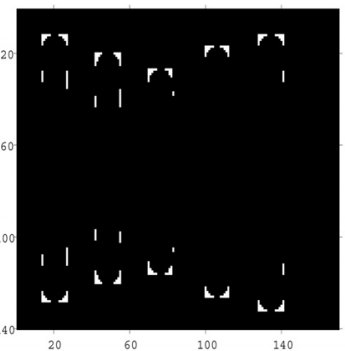

(b)

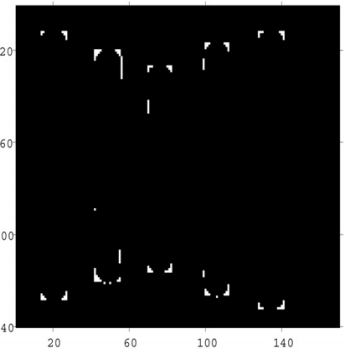

(c)

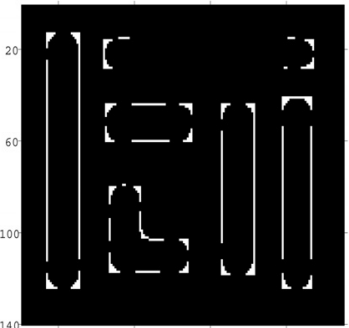

$(d)$

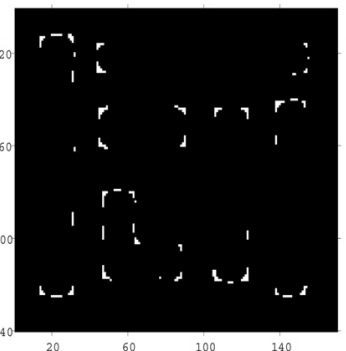

(e)

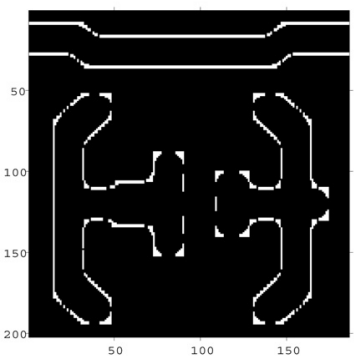

(f)
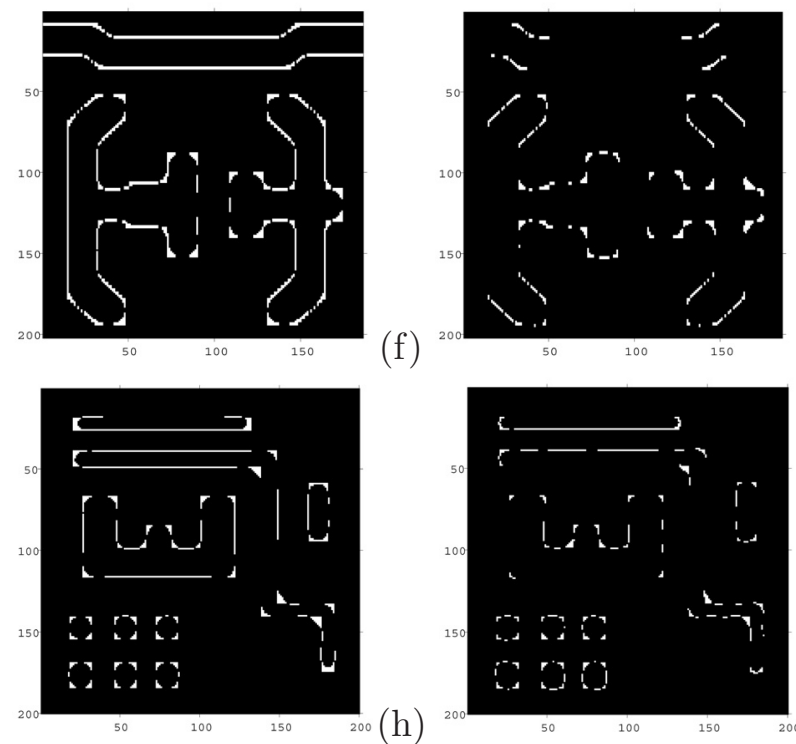

(g)

(h)

Figure 7. Edge placement error map.

the corner-rounding effect in the output patterns is significant. On the other hand, the synthesized masks with some small and isolated assist features (see Figures 5(d) and 6(d)) are automatically generated using our algorithm. Similar to the previous examples, the optimal mask achieves satisfactory output patterns where the corners of the rectangular bars are well preserved (see also Figure 7 for more details). In these two cases, the pattern errors are apparently smaller using our optimal masks as input, as can be seen in Table 1 .

To further evaluate the robustness of the proposed method, we compute the edge placement error (EPE) map, which measures the difference between the output pattern and the target pattern. Each EPE map shown below is generated by accumulating multiple EPE maps across 
the defocus range $[0 \mathrm{~nm}, 300 \mathrm{~nm}]$. The EPE map indicates the level of deviation between the printed edge and the target edge from infocus to defocus. The more robust the mask pattern is, the smaller the observed deviation is. Figure 7 displays the EPE maps of the previous patterns when the inputs are the target patterns (left column) and the optimal masks (right column), respectively. As can be seen, the accumulated edge placement errors occur mostly at the end of long bars and pattern corners, which in optical lithography are the critical locations that are sensitive to optical proximity effects and process variations. In these examples, our results show that the optimal masks produce less deviation, especially at the line ends and corners under different focus conditions, and therefore the proposed method can effectively enhance mask robustness.

Generally speaking, these results show that without mask optimization, using the target pattern as an input directly will lead to unsatisfactory pattern fidelity. On the other hand, the optimal mask synthesized by our method gives a very good output pattern contour with a relatively small error and our algorithm is also robust to focus fluctuation.

6.2. Impact of global complexity penalty. In this subsection, we discuss the impact of the global complexity penalty term on the optimal mask and the pattern fidelity by choosing different values of $\lambda_{2}$. Figure 8 shows the optimal masks of Figures 4-6 for $\lambda_{2}=0$ (left column), $\lambda_{2}=0.4$ (middle column), and $\lambda_{2}=0.8$ (right column). As can be seen, when the global complexity penalty term is neglected (i.e., $\lambda_{2}=0$ ), some small, irregular, and isolated assist features are automatically generated using our algorithm. But when $\lambda_{2}$ becomes larger, most of the assist features are suppressed, and the optimal mask resembles the target pattern when $\lambda_{2}=0.8$. Theoretically, the optimal mask would be equal to the target pattern as $\lambda_{2} \rightarrow \infty$. This phenomenon is expected and is consistent with our analysis and discussion in section 4 . Table 2 reports the corresponding pattern errors and percentage errors. As far as the pattern fidelity is concerned, the best results are achieved when $\lambda_{2}=0$, and the pattern errors slightly increase when $\lambda_{2}=0.4$. But when $\lambda_{2}=0.8$, some useful assist features are removed, leading to poor pattern fidelity. It is important to note that while $\lambda_{2}=0$ gives the smallest pattern errors, the corresponding optimal masks are a bit complex compared with the ones when $\lambda_{2}=0.4$ and $\lambda_{2}=0.8$. Our results suggest that there is a tradeoff between the mask complexity and the pattern fidelity, and, therefore, one can always obtain a less complex mask at the expense of the pattern fidelity by choosing a suitable value of $\lambda_{2}$. In these three cases, if both the mask complexity and pattern fidelity are taken into consideration, the best results perhaps correspond to the cases when $\lambda_{2}=0.4$. Generally speaking, these results clearly indicate the importance of the global complexity penalty and reveal that our proposed variational formulation could be used to synthesize a simple mask with a small pattern error where the complexity of the optimal mask could be adjusted arbitrarily. We believe that this property would be an important and welcome advantage if it could be used in the semiconductor manufacturing process.

6.3. Comparative results. We compare our methodology with the machine learning approach [14]. In this method, the authors proposed minimizing the weighted pattern error with focus variation, where the weights are assumed to be zero-mean Gaussian distributed with standard deviation $150 \mathrm{~nm}$. The optimization problem was then solved by the gradient descent algorithm with initial mask $U^{\text {init }}=0.1 I_{0}+0.45$. In the following experiments, the

Copyright (c) by SIAM. Unauthorized reproduction of this article is prohibited. 


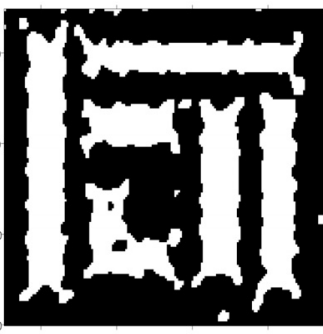

(a)

(d)

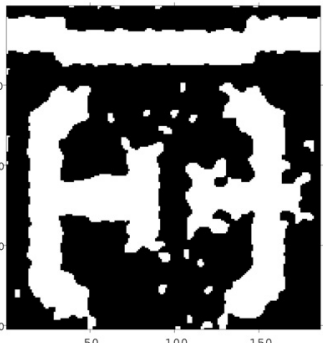

(g)

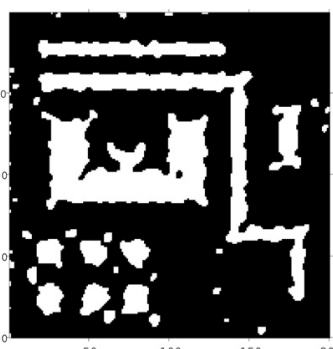

(b)
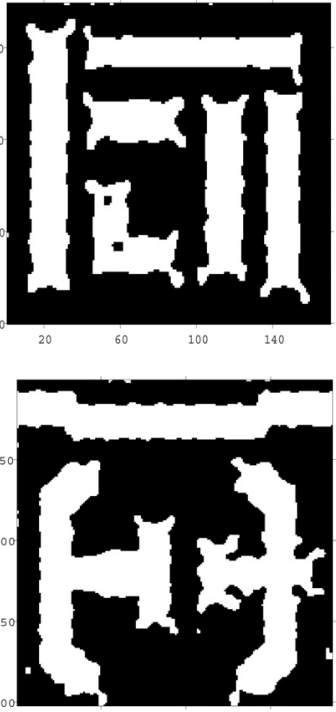

(e)

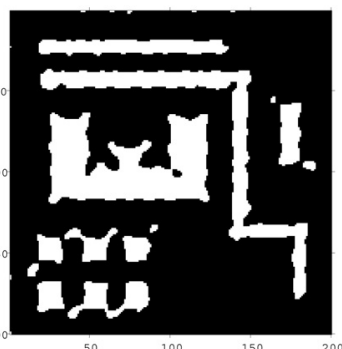

(c)
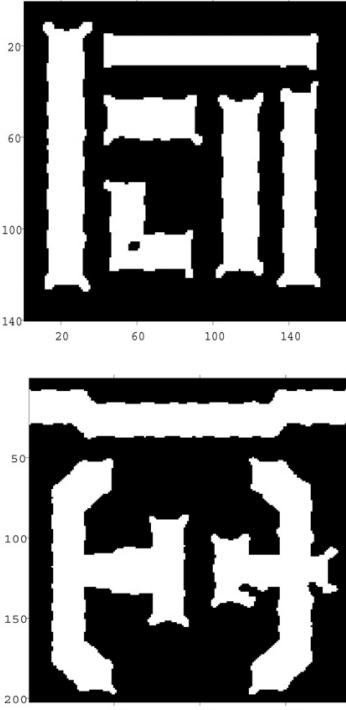

(f)

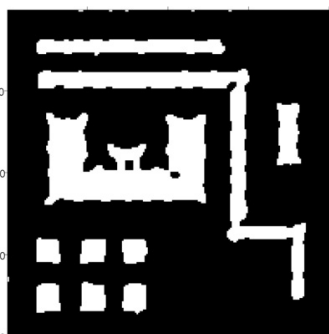

Figure 8. The optimal masks of Figures 4-6 for different values of $\lambda_{2}$. From the left column to the right column, the values of $\lambda_{2}$ are $0,0.4$, and 0.8 , respectively.

Table 2

Pattern errors and the corresponding percentage errors of Figures 4-6 at the best focus and under defocus $300 \mathrm{~nm}$ for different values of $\lambda_{2}$.

\begin{tabular}{c||cc|cc|cc}
\hline \hline \multirow{2}{*}{ Figure } & \multicolumn{2}{|c|}{$\lambda_{2}=0$} & \multicolumn{2}{c|}{$\lambda_{2}=0.4$} & \multicolumn{2}{c}{$\lambda_{2}=0.8$} \\
\cline { 2 - 7 } & $\beta=0$ & $\beta=300$ & $\beta=0$ & $\beta=300$ & $\beta=0$ & $\beta=300$ \\
\hline 4 & 47 & 256 & 62 & 272 & 121 & 345 \\
& $(0.20 \%)$ & $(1.08 \%)$ & $(0.26 \%)$ & $(1.14 \%)$ & $(0.51 \%)$ & $(1.45 \%)$ \\
\hline 5 & 228 & 432 & 247 & 464 & 305 & 524 \\
& $(0.61 \%)$ & $(1.15 \%)$ & $(0.66 \%)$ & $(1.23 \%)$ & $(0.81 \%)$ & $(1.39 \%)$ \\
\hline 6 & 273 & 636 & 275 & 648 & 378 & 725 \\
& $(0.68 \%)$ & $(1.59 \%)$ & $(0.69 \%)$ & $(1.62 \%)$ & $(0.95 \%)$ & $(1.81 \%)$ \\
\hline \hline
\end{tabular}

same parameter setting (except the mask initialization) is used as in [14]. We shall assess the performance of our method and the machine learning approach by comparing their optimal masks and the corresponding output patterns. In addition, we shall also compare the results of the proposed method by using different initialization strategies (see the last two rows of Figures 9-11).

Copyright (C) by SIAM. Unauthorized reproduction of this article is prohibited. 
(a)

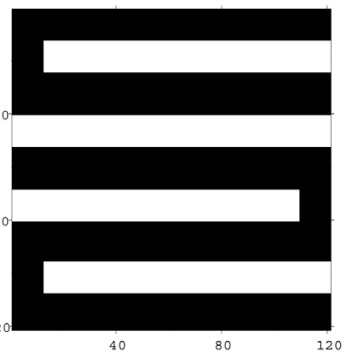

(b)

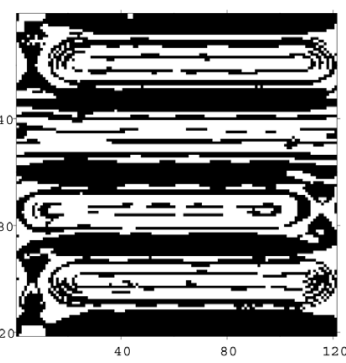

${ }_{120}(\mathrm{c})$

(e)

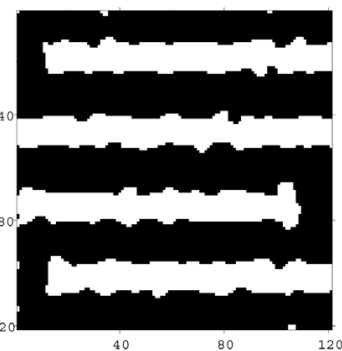

$20(f)$
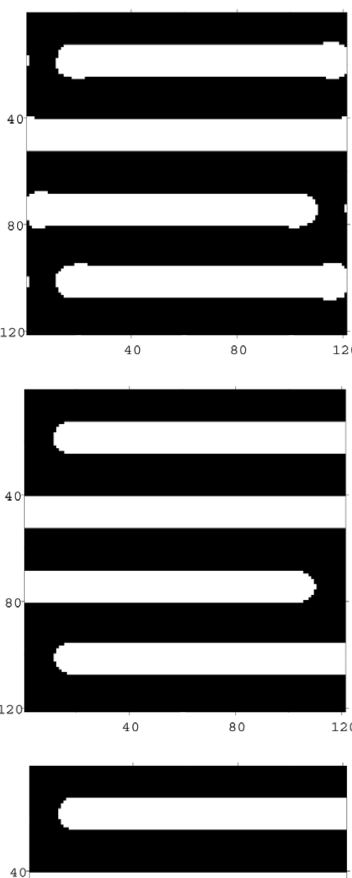

(h)

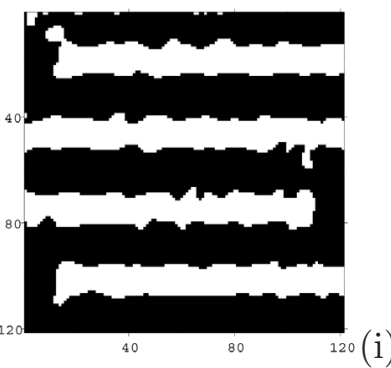

$120(\mathrm{~g})$
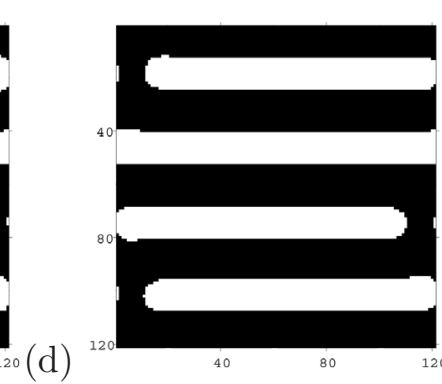

d)
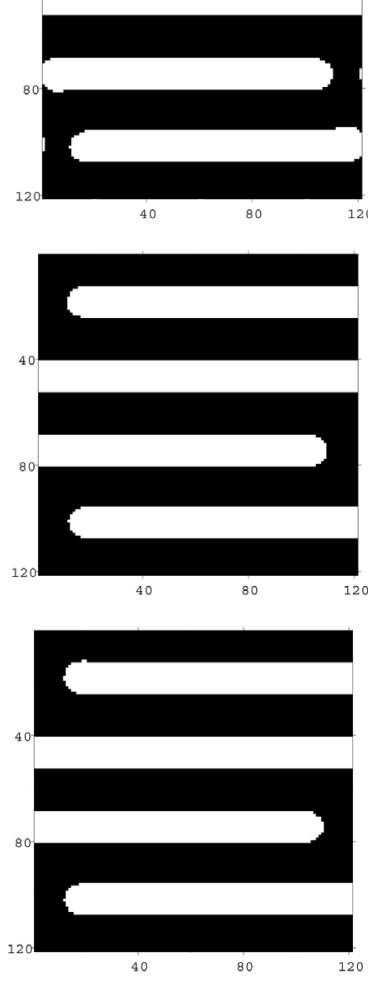

Figure 9. (a) Target pattern of size $121 \times 121$ pixels. (b) Optimal mask using the method in [14]. (c)-(d) Output patterns at the best focus and under defocus $250 \mathrm{~nm}$ with (b) as input. (e) Optimal mask generated by our method with $U^{\text {init }}=U D$. (f)-(g) Output patterns at the best focus and under defocus 250nm with (e) as input. (h) Optimal mask generated by our method with $U^{\text {init }}=0.5 I_{0}+\mathcal{N}(0,1)$. (i)-(j) Output patterns at the best focus and under defocus 250nm with (h) as input.

Figures 9-11 show the comparative results of optimal masks and the associated output patterns. For all cases, one first observes that the optimal masks generated by the machine learning approach (see Figures 9(b), 10(b), 11(b)) are rather complex compared with the optimal masks synthesized by our method (see Figures 9(e), 9(h), 10(e), 10(h), 11(e), 11(h)). This may be explained by the fact that the machine learning approach does not take into 
(a)

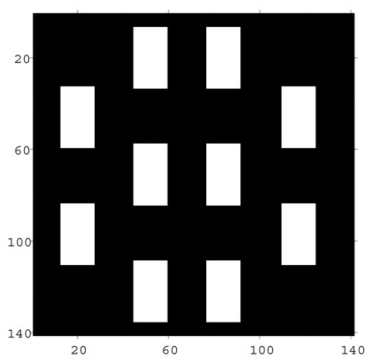

(b)

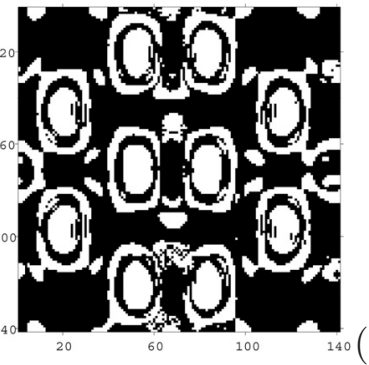

(e)

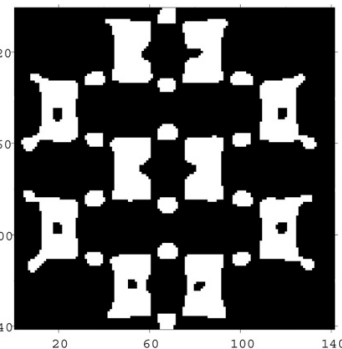

(f)

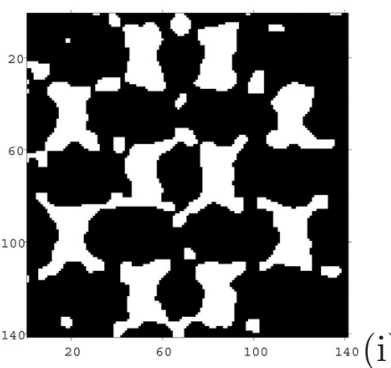

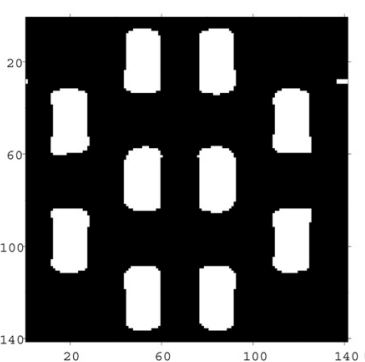

(d)
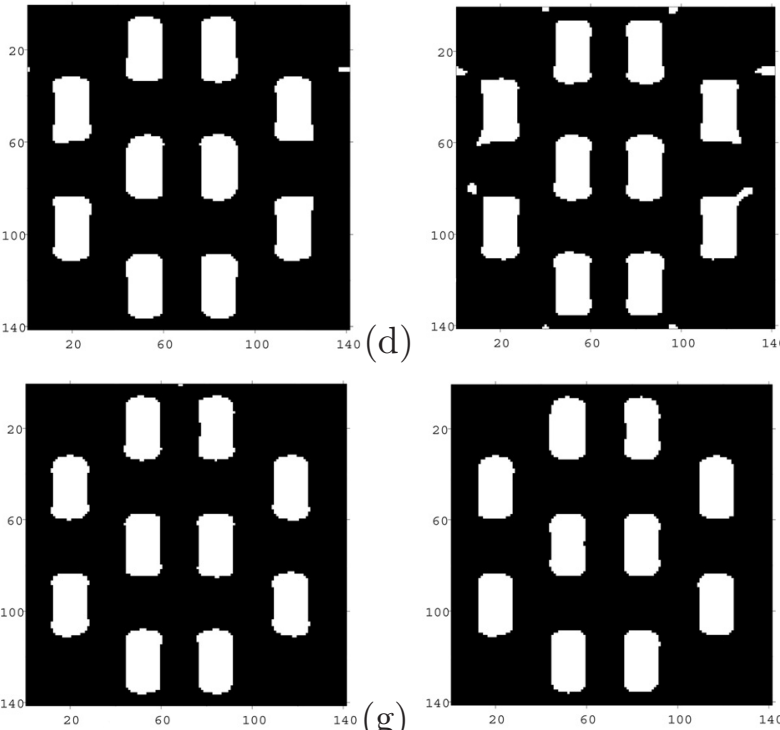

$0(\mathrm{~g})$
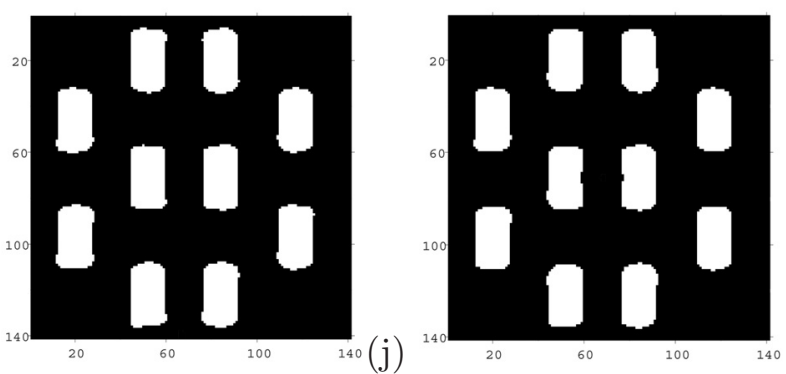

Figure 10. (a) Target pattern of size $141 \times 141$ pixels. (b) Optimal mask using the method in [14]. (c)-(d) Output patterns at the best focus and under defocus 250nm with (b) as input. (e) Optimal mask generated by our method with $U^{\text {init }}=U D$. (f)-(g) Output patterns at the best focus and under defocus 250nm with (e) as input. (h) Optimal mask generated by our method with $U^{\text {init }}=0.5 I_{0}+\mathcal{N}(0,1)$. (i)-(j) Output patterns at the best focus and under defocus $250 \mathrm{~nm}$ with (h) as input.

account the mask complexity, whereas our method incorporates penalty terms that can control the mask structure. Note that we have set $\lambda_{2}=0$ and $\lambda_{3}=1.0$ for Figures $9-10$ and thus we shall control their mask complexity only locally. Nevertheless, we have found that setting $\lambda_{2}=0$ is good enough to achieve low complexity and high quality masks with small pattern error in these two cases. For Figure 11, we have set $\lambda_{2}=0.4$ and $\lambda_{3}=1.0$, and hence both 
(a)

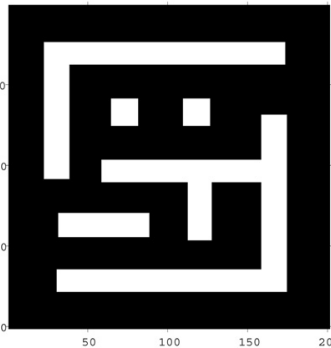

(b)

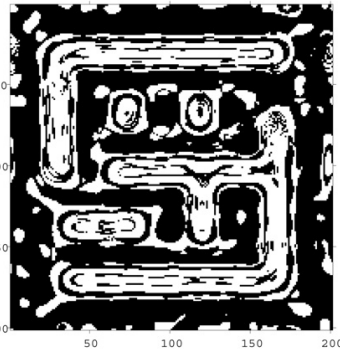

$200(c)$

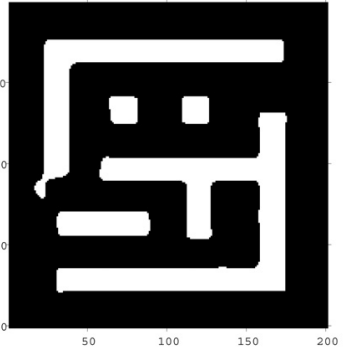

$(d)$

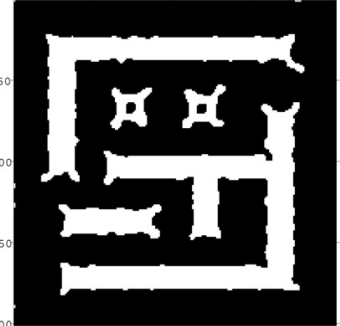

(e)

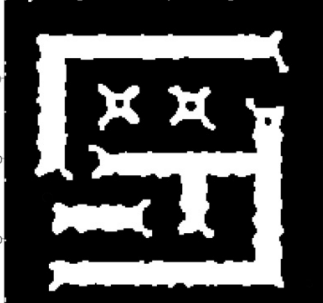

(h)

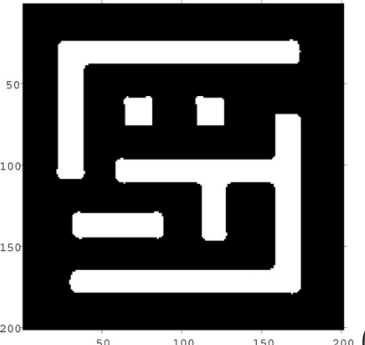

$(\mathrm{g})$

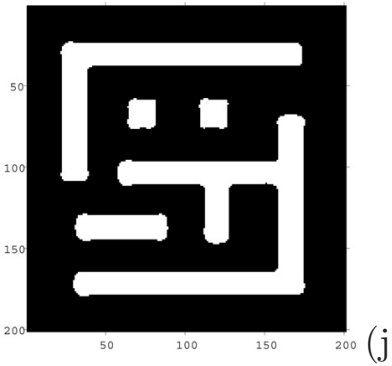

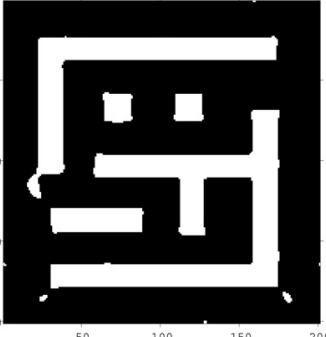
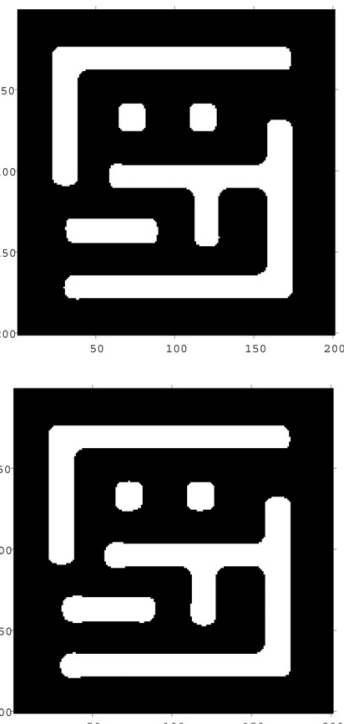

Figure 11. (a) Target pattern of size $201 \times 201$ pixels. (b) Optimal mask using the method in [14]. (c)-(d) Output patterns at the best focus and under defocus 250nm with (b) as input. (e) Optimal mask generated by our method with $U^{\text {init }}=U D$. (f)-(g) Output patterns at the best focus and under defocus 250nm with (e) as input. (h) Optimal mask generated by our method with $U^{\text {init }}=0.5 I_{0}+\mathcal{N}(0,1)$. (i)-(j) Output patterns at the best focus and under defocus 250nm with (h) as input.

global and local mask structures are taken into consideration. The output patterns at the best focus and under defocus $250 \mathrm{~nm}$ are also shown in the corresponding figures. We see that parts of the output patterns for the machine learning approach are not accurately reproduced, whereas our method gives very good pattern contours. The summary of pattern errors (and percentage errors) is reported in Table 3. In terms of pattern fidelity, our algorithm in general 
Table 3

Pattern errors and the corresponding percentage errors of the machine learning approach [14] and our method at the best focus and under defocus 250nm. Proposed method 1: Our method with uniform distribution as initial mask $\left(U^{\text {init }}=U D\right)$. Proposed method 2: Our method with $U^{\text {init }}=0.5 I_{0}+\mathcal{N}(0,1)$.

\begin{tabular}{c||cc|cc|cc}
\hline \hline \multirow{2}{*}{ Figure } & \multicolumn{2}{|c|}{ Method in [14] } & \multicolumn{2}{|c}{ Proposed method 1 } & \multicolumn{2}{c}{ Proposed method 2 } \\
\cline { 2 - 7 } & $\beta=0$ & $\beta=250$ & $\beta=0$ & $\beta=250$ & $\beta=0$ & $\beta=250$ \\
\hline 9 & 85 & 103 & 43 & 63 & 44 & 65 \\
& $(0.58 \%)$ & $(0.70 \%)$ & $(0.29 \%)$ & $(0.43 \%)$ & $(0.30 \%)$ & $(0.44 \%)$ \\
\hline 10 & 289 & 338 & 205 & 227 & 221 & 245 \\
& $(1.45 \%)$ & $(1.70 \%)$ & $(1.03 \%)$ & $(1.14 \%)$ & $(1.11 \%)$ & $(1.23 \%)$ \\
\hline 11 & 202 & 259 & 134 & 189 & 154 & 211 \\
& $(0.50 \%)$ & $(0.64 \%)$ & $(0.33 \%)$ & $(0.47 \%)$ & $(0.38 \%)$ & $(0.52 \%)$ \\
\hline \hline
\end{tabular}

outperforms the machine learning approach.

In addition to the comparisons of our method and the machine learning approach, we also compare the optimal masks and the pattern fidelity of the proposed method when different initialization strategies are adopted. Note that for all of the above experiments, the initial mask is generated based on the uniform distribution (i.e., $U^{\text {init }}=U D$ ). Here, we shall adopt another initialization $U^{\text {init }}=0.5 I_{0}+\mathcal{N}(0,1)$, where $\mathcal{N}(0,1)$ is the zero-mean Gaussian noise with variance 1. Figures $9(\mathrm{e}), 10(\mathrm{e})$, and $11(\mathrm{e})$ show the optimal masks with $U^{\text {init }}=U D$, whereas Figures $9(\mathrm{~h}), 10(\mathrm{~h})$, and 11(h) display the optimal masks with $U^{\text {init }}=0.5 I_{0}+\mathcal{N}(0,1)$. Their output patterns are shown in the same row. As can be seen, both initializations give similar optimal masks in Figures 9 and 11. On the other hand, we observe that the optimal mask in Figure 10(e) looks more regular than the one in Figure 10(h). Table 3 reports the corresponding pattern errors and percentage errors. While both initializations result in similar mask structures, our study indicates that using $U^{\text {init }}=U D$ as an initialization gives smaller pattern errors compared with using $U^{i n i t}=0.5 I_{0}+\mathcal{N}(0,1)$. Interestingly, we also found that using $U^{\text {init }}=U D$ as an initial guess in our algorithm always outperforms any other initializations in the form of a linear combination of target pattern.

7. Extensions and conclusions. In this section, we shall discuss the extension of our proposed framework to partially coherent imaging systems, which are currently adopted in the semiconductor manufacturing industry. We then conclude with some discussions.

7.1. Extensions to partially coherent imaging systems. Practical lithography systems in real-world industrial simulations are partially coherent imaging systems. Therefore, the proposed framework needs to be extended to apply to such systems in order to have practical value to the lithography community. Essentially, the major issue in our formulation is how to obtain the aerial image in a partially coherent imaging system. Here, we shall only discuss the computation of the aerial image without focus variation. Incorporating the focus variation into the aerial image is straightforward.

The aerial image under partially coherent imaging based on the Hopkin approach [1] can be computed as

$$
I^{A}(x)=\int_{-\infty}^{\infty} \int_{-\infty}^{\infty} \int_{-\infty}^{\infty} J(v) h\left(v_{1}+v\right) h^{\dagger}\left(v_{2}+v\right) u\left(v_{1}\right) u^{\dagger}\left(v_{2}\right) e^{-j 2 \pi\left(v_{1}-v_{2}\right) x} d v_{1} d v_{2} d v
$$

Copyright (c) by SIAM. Unauthorized reproduction of this article is prohibited. 
Here, $J$ and $u$ are the functions of the effective source and the mask spectrum, respectively. Note that the aerial image is formed by adding the interferences of wave pairs traveling from directions corresponding to $v_{1}$ and $v_{2}$. The interaction between each wave pair is weighted by a factor known as the transmission cross-coefficient $(T C C)$ :

$$
T C C\left(v_{1}, v_{2}\right)=\int_{-\infty}^{\infty} J(v) h\left(v_{1}+v\right) h^{\dagger}\left(v_{2}+v\right) d v .
$$

Incorporating the $T C C$ into the above aerial image function leads to

$$
I^{A}(x)=\int_{-\infty}^{\infty} \int_{-\infty}^{\infty} T C C\left(v_{1}, v_{2}\right) u\left(v_{1}\right) u^{\dagger}\left(v_{2}\right) e^{-j 2 \pi\left(v_{1}-v_{2}\right) x} d v_{1} d v_{2} .
$$

To improve efficiency, the TCC can be decomposed into a series of eigenvectors $\phi_{l}$ and the corresponding eigenvalues $\widetilde{\lambda}_{l}[10,32,39]$ as

$$
\operatorname{TCC}\left(v_{1}, v_{2}\right)=\sum_{l=1}^{\infty} \widetilde{\lambda}_{l} \phi_{l}\left(v_{1}\right) \phi_{l}^{\dagger}\left(v_{2}\right) .
$$

For typical exposure systems, the magnitudes of a few eigenvalues are much larger than the rest. Assuming that there are $L$ dominant eigenvectors, we can thus approximate the $T C C$ as

$$
\operatorname{TCC}\left(v_{1}, v_{2}\right) \approx \sum_{l=1}^{L} \tilde{\lambda}_{l} \phi_{l}\left(v_{1}\right) \phi_{l}^{\dagger}\left(v_{2}\right) .
$$

Then the aerial image can be calculated as

$$
\begin{aligned}
I^{A}(x) & =\sum_{l=1}^{L} \tilde{\lambda_{l}} \int_{-\infty}^{\infty} \int_{-\infty}^{\infty} \phi_{l}\left(v_{1}\right) \phi_{l}^{\dagger}\left(v_{2}\right) u\left(v_{1}\right) u^{\dagger}\left(v_{2}\right) e^{-j 2 \pi\left(v_{1}-v_{2}\right) x} d v_{1} d v_{2} \\
& =\sum_{l=1}^{L} \tilde{\lambda_{l}}\left|\int_{-\infty}^{\infty} \phi_{l}\left(v_{1}\right) u\left(v_{1}\right) e^{-j 2 \pi v_{1} x} d v_{1}\right|^{2} \\
& =\sum_{l=1}^{L} \tilde{\lambda}_{l}\left|\left(\Phi_{l} * U\right)(x)\right|^{2},
\end{aligned}
$$

where $\Phi_{l}$ is the inverse Fourier transform of $\phi_{l}$. Therefore, the aerial image in the partially coherent imaging system can be formulated as the superposition of a finite number of coherent images. Once the aerial image is computed using (7.1), the output pattern is obtained by taking the sigmoid function of the aerial image. The corresponding energy functional can also be defined in a similar manner (see (4.1)), and the optimization problem under the partially coherent imaging system can be solved directly using our proposed mask synthesis algorithm.

7.2. Conclusions. This paper presents a variational formulation and a computational algorithm for pixel-based binary photomask design using inverse lithography. Compared with the classical and current state-of-the-art functionals, our proposed formulation is robust to process variations and can make the resulting mask less complex. We have also proved the

Copyright (C) by SIAM. Unauthorized reproduction of this article is prohibited. 
existence of a minimizer of the proposed functional. To optimize the proposed functional, we present an effective alternating minimization scheme and adopt the fast duality projection algorithm of Chambolle. Experimental results reveal that our methodology can effectively synthesize robust photomasks compared with some existing approaches.

While the mask synthesis algorithm presented in this paper achieves promising results in computational lithography, we apply the proposed algorithm only to coherent imaging systems. Extensive numerical experiments should be performed in the partially coherent imaging system to show the effectiveness of our methodology. In addition, an intervention scheme [17], which prevents undesirable geometrical characteristics of mask features, can also be incorporated into our framework. These issues will be explored in our future work.

Appendix A. We shall show that if $\tilde{U}$ is a minimizer of $\widetilde{Q}_{\beta}$ defined in (5.4), then

$$
\frac{\partial \widetilde{Q}_{\beta}}{\partial \tilde{U}}=\sum_{i=1}^{N} \eta_{i} g_{\beta_{i}}(\tilde{U})=0
$$

where $g_{\beta_{i}}(\tilde{U})$ is defined by $(5.7)$.

We begin by computing the partial derivatives of the sigmoid function $\operatorname{sig}(z)=1 /(1+$ $\exp (-a(z-t r)))$ and the convolution $\left(H_{\beta_{i}} * U\right)(z)$. It is straightforward to show that

$$
\begin{gathered}
\frac{\partial \operatorname{sig}(z)}{\partial z}=a \cdot \operatorname{sig}(z)(1-\operatorname{sig}(z)) \\
\frac{\partial\left(H_{\beta_{i}} * U\right)(z)}{\partial U(p)}=\frac{\partial \int_{\Omega} H_{\beta_{i}}(z-v) U(v) d v}{\partial U(p)}=H_{\beta_{i}}(z-p) .
\end{gathered}
$$

Using the above equations, the partial derivatives of the aerial image $I_{\beta_{i}}^{A}(x)=\left|\left(H_{\beta_{i}} * U\right)(x)\right|^{2}$ and the output image $I_{\beta_{i}}(x)=\operatorname{sig}\left(I_{\beta_{i}}^{A}(x)\right)$ are given by

$$
\begin{aligned}
\frac{\partial I_{\beta_{i}}^{A}(x)}{\partial U(p)}= & \left(H_{\beta_{i}}^{\dagger} * U\right)(x) H_{\beta_{i}}(x-p)+\left(H_{\beta_{i}} * U\right)(x) H_{\beta_{i}}^{\dagger}(x-p), \\
\frac{\partial I_{\beta_{i}}(x)}{\partial U(p)}= & a \cdot I_{\beta_{i}}(x)\left(1-I_{\beta_{i}}(x)\right)\left(\left(H_{\beta_{i}}^{\dagger} * U\right)(x) H_{\beta_{i}}(x-p)\right. \\
& \left.+\left(H_{\beta_{i}} * U\right)(x) H_{\beta_{i}}^{\dagger}(x-p)\right),
\end{aligned}
$$

where $H_{\beta_{i}}^{\dagger}$ is a complex conjugate of $H_{\beta_{i}}$. In the following, we shall simply drop the argument $x \in \Omega$ whenever there is no ambiguity.

Given the energy functional defined in (5.4) and using (A.1) and (A.2), the partial deriv- 
ative of $\widetilde{Q}_{\beta}$ with respect to $U$ is

$$
\begin{aligned}
\frac{\partial \widetilde{Q}_{\beta}}{\partial U}= & \sum_{i=1}^{N} \eta_{i}\left\{\lambda_{1} \int_{\Omega}\left(I_{\beta_{i}}-I_{0}\right)\left(\frac{\partial I_{\beta_{i}}}{\partial U}\right) d x+\lambda_{2}\left(U-I_{0}\right)+\frac{1}{\theta_{1}}(U-V)\right. \\
& \left.+\frac{1}{\theta_{2}} \int_{\Omega}\left(I_{\beta_{i}}^{A}-J_{\beta_{i}}^{A}\right)\left(\frac{\partial I_{\beta_{i}}^{A}}{\partial U}\right) d x+\lambda_{5}(2-4 U)\right\}, \\
= & \sum_{i=1}^{N} \eta_{i}\left\{\lambda _ { 1 } \int _ { \Omega } a \cdot ( I _ { \beta _ { i } } - I _ { 0 } ) I _ { \beta _ { i } } ( 1 - I _ { \beta _ { i } } ) \left(\left(H_{\beta_{i}}^{\dagger} * U\right) H_{\beta_{i}}(x-p)\right.\right. \\
& \left.+\left(H_{\beta_{i}} * U\right) H_{\beta_{i}}^{\dagger}(x-p)\right) d x+\lambda_{2}\left(U-I_{0}\right)+\frac{1}{\theta_{1}}(U-V) \\
& +\frac{1}{\theta_{2}} \int_{\Omega}\left(I_{\beta_{i}}^{A}-J_{\beta_{i}}^{A}\right)\left(\left(H_{\beta_{i}}^{\dagger} * U\right) H_{\beta_{i}}(x-p)+\left(H_{\beta_{i}} * U\right) H_{\beta_{i}}^{\dagger}(x-p)\right) d x \\
& \left.+\lambda_{5}(2-4 U)\right\}, \\
= & \sum_{i=1}^{N} \eta_{i}\left\{a \lambda_{1} H_{\beta_{i}} *\left(I_{\beta_{i}} \bullet\left(1-I_{\beta_{i}}\right) \bullet\left(I_{\beta_{i}}-I_{0}\right) \bullet\left(H_{\beta_{i}}^{\dagger} * U\right)\right)\right. \\
& +a \lambda_{1} H_{\beta_{i}}^{\dagger} *\left(I_{\beta_{i}} \bullet\left(1-I_{\beta_{i}}\right) \bullet\left(I_{\beta_{i}}-I_{0}\right) \bullet\left(H_{\beta_{i}} * U\right)\right) \\
& +\frac{1}{\theta_{2}} \cdot H_{\beta_{i}} *\left(\left(I_{\beta_{i}}^{A}-J_{\beta_{i}}^{A}\right) \bullet\left(H_{\beta_{i}}^{\dagger} * U\right)\right)+\frac{1}{\theta_{2}} \cdot H_{\beta_{i}}^{\dagger} *\left(\left(I_{\beta_{i}}^{A}-J_{\beta_{i}}^{A}\right) \bullet\left(H_{\beta_{i}} * U\right)\right) \\
& \left.+\frac{1}{\theta_{1}}(U-V)+\lambda_{2}\left(U-I_{0}\right)+\lambda_{5}(2-4 U)\right\} \cdot
\end{aligned}
$$

Let $\Im_{i}^{1}=a \lambda_{1} I_{\beta_{i}} \bullet\left(1-I_{\beta_{i}}\right) \bullet\left(I_{\beta_{i}}-I_{0}\right)+\frac{1}{\theta_{2}}\left(I_{\beta_{i}}^{A}-J_{\beta_{i}}^{A}\right)$. Then we obtain

$$
\begin{aligned}
\frac{\partial \widetilde{Q}_{\beta}}{\partial U}= & \sum_{i=1}^{N} \eta_{i}\left\{H_{\beta_{i}} *\left(\left(H_{\beta_{i}}^{\dagger} * U\right) \bullet \Im_{i}^{1}\right)+H_{\beta_{i}}^{\dagger} *\left(\left(H_{\beta_{i}} * U\right) \bullet \Im_{i}^{1}\right)\right. \\
& \left.+\left(\frac{1}{\theta_{1}}(U-V)+\lambda_{2}\left(U-I_{0}\right)+\lambda_{5}(2-4 U)\right)\right\} .
\end{aligned}
$$

Substitute $\tilde{U}$ into (A.3) and set it to zero; the result follows.

\section{REFERENCES}

[1] M. Born and E. Wolf, Principles of Optics, Cambridge University Press, Cambridge, UK, 1999.

[2] A. Chambolle, An algorithm for total variation minimization and applications, J. Math. Imaging Vision, 20 (2004), pp. 89-97.

[3] S. H. Chan AND E. Y. LAM, Inverse image problem of designing phase shifting masks in optical lithography, in Proceedings of the IEEE International Conference on Image Processing (ICIP), 2008, pp. $1832-1835$.

[4] S. H. Chan, A. K. Wong, And E. Y. Lam, Initialization for robust inverse synthesis of phase-shifting masks in optical projection lithography, Opt. Express, 16 (2008), pp. 14746-14760.

Copyright $($ b by SIAM. Unauthorized reproduction of this article is prohibited. 
[5] T. F. Chan And K. Chen, An optimization-based multilevel algorithm for total variation image denoising, Multiscale Model. Simul., 5 (2006), pp. 615-645.

[6] J. F. Chen, T. Laidig, K. E. Wampler, and R. Caldwell, Optical proximity correction for intermediate-pitch features using sub-resolution scattering bars, J. Vac. Sci. Technol. B, 15 (1997), pp. 2426-2433.

[7] S. K. Choy, M. L. TAng, And C. S. Tong, Image segmentation using fuzzy region competition and spatial/frequency information, IEEE Trans. Image Process., 20 (2011), pp. 1473-1484.

[8] N. CobB And A. ZAKHor, Fast, low-complexity mask design, in Optical/Laser Microlithography VIII, Proceedings of SPIE, Vol. 2440, 1995, pp. 313-327.

[9] A. Erdmann, T. Fühner, T. Schnattinger, and B. Tollkühn, Toward automatic mask and source optimization for optical lithography, in Optical Microlithography XVII, Proceedings of SPIE, Vol. 5377, 2004, pp. 646-657.

[10] H. Gamo, Matrix treatment of partial coherence, in Progress in Optics, Vol. 3, North-Holland, Amsterdam, 1964, pp. 187-332.

[11] Y. GRANIK, Solving inverse problems of optical microlithography, in Optical Microlithography XVIII, Proceedings of SPIE, Vol. 5754, 2005, pp. 506-526.

[12] Y. Granik, Fast pixel-based mask optimization for inverse lithography, J. Microlith. Microfab. Microsyst., 5 (2006), 043002.

[13] W. Huang, C. Lin, C. Kuo, C. Huang, J. Lin, J. Chen, R. Liu, Y. Ku, and B. Lin, Two threshold resist models for optical proximity correction, in Optical Microlithography XVII, Proceedings of SPIE, Vol. 5377, 2004, pp. 1536-1543.

[14] N. JiA AND E. Y. LAM, Machine learning for inverse lithography: Using stochastic gradient descent for robust photomask synthesis, J. Opt., 12 (2010), 045601.

[15] N. JiA AND E. Y. LAm, Pixelated source mask optimization for process robustness in optical lithography, Opt. Express, 19 (2011), pp. 19384-19398.

[16] N. JiA, A. K. Wong, AND E. Y. LAM, Robust mask design with defocus variation using inverse synthesis, in Lithography Asia 2008, Proceedings of SPIE, Vol. 7140, 2008, 71401W.

[17] N. Jia, A. K. Wong, And E. Y. LAm, Regularization of inverse photomask synthesis to enhance manufacturability, in Lithography Asia 2009, Proceedings of SPIE, Vol. 7520, 2009, 75200E.

[18] E. Y. LAm AND A. K. WOnG, Computation lithography: Virtual reality and virtual virtuality, Opt. Express, 17 (2009), pp. 12259-12268.

[19] E. Y. LAm And A. K. Wong, Nebulous hotspot and algorithm variability in computation lithography, J. Micro/Nanolith. MEMS MOEMS, 9 (2010), 033002.

[20] F. Li AND M. NG, Kernel density estimation based multiphase fuzzy region competition method for texture image segmentation, Commun. Comput. Phys., 8 (2010), pp. 623-641.

[21] Y. LiU AND A. ZAKHor, Binary and phase shifting mask design for optical lithography, IEEE Trans. Semicond. Manuf., 5 (1992), pp. 138-151.

[22] X. Ma And G. R. ARCE, Generalized inverse lithography methods for phase-shifting mask design, Opt. Express, 15 (2007), pp. 15066-15079.

[23] X. Ma And G. R. Arce, Binary mask optimization for inverse lithography with partially coherent illumination, J. Opt. Soc. Amer. A, 25 (2008), pp. 2960-2970.

[24] X. MA AND G. R. ARCE, Binary mask optimization for forward lithography based on the boundary layer model in coherent systems, J. Opt. Soc. Amer. A, 26 (2009), pp. 1687-1695.

[25] C. MAcK, Fundamental Principles of Optical Lithography: The Science of Microfabrication, John Wiley \& Sons, West Sussex, UK, 2007.

[26] B. Mory And R. Ardon, Fuzzy region competition: A convex two-phase segmentation framework, in Scale Space and Variational Methods in Computer Vision, Lecture Notes in Comput. Sci. 4485, Springer-Verlag, Berlin, Heidelberg, 2007, pp. 214-226.

[27] M. NG, L. QI, Y. YANG, AND Y. HuAng, On semismooth Newton's methods for total variation minimization, J. Math. Imaging Vision, 27 (2007), pp. 265-276.

[28] Y. ОH, J. C. LEE, AND S. Lim, Resolution enhancement through optical proximity correction and stepper parameter optimization for 0.12- $\mu \mathrm{m}$ mask pattern, in Optical Microlithography XII, Proceedings of SPIE, Vol. 3679, 1999, pp. 607-613.

Copyright (C) by SIAM. Unauthorized reproduction of this article is prohibited. 
[29] O. W. Otto, J. G. Garofalo, K. K. Low, C. Yuan, R. C. Henderson, C. Pierrat, R. L. KosteLAK, S. VAIDYA, AND P. K. VASUdEV, Automated optical proximity correction: A rules-based approach, in Optical/Laser Microlithography VII, Proceedings of SPIE, Vol. 2197, 1994, pp. 278-293.

[30] L. PAng, Y. LiU, And D. ABrams, Inverse lithography technology (ILT): A natural solution for modelbased SRAF at $45 \mathrm{~nm}$ and $32 \mathrm{~nm}$, in Photomask and Next-Generation Lithography Mask Technology XIV, Proceedings of the SPIE, Vol. 6607, 2007, 660739.

[31] V. Pati and T. Kailath, Phase-shifting masks for microlithography: Automated design and mask requirements, J. Opt. Soc. Amer. A, 11 (1994), pp. 2438-2452.

[32] Y. C. Pati, A. A. Ghazanfarian, and R. F. Pease, Exploiting structure in fast aerial image computation for integrated circuit patterns, IEEE Trans. Semicond. Manuf., 10 (1997), pp. 62-74.

[33] A. Poonawala And P. Milanfar, OPC and PSM design using inverse lithography: A non-linear optimization approach, in Optical Microlithography XIX, Proceedings of SPIE, Vol. 6154, 2006, pp. 1159-1172.

[34] A. Poonawala and P. Milanfar, Double-exposure mask synthesis using inverse lithography, J. Micro/ Nanolith. MEMS MOEMS, 6 (2007), 043001.

[35] A. Poonawala and P. Milanfar, Mask design for optical microlithography - An inverse imaging problem, IEEE Trans. Image Process., 16 (2007), pp. 774-788.

[36] A. Poonawala, B. Painter, And J. Mayhew, Model-based assist feature placement: An inverse imaging approach, in Photomask Technology 2008, Proceedings of SPIE, Vol. 7122, 2008, 71220U.

[37] J. Randall, K. Ronse, T. Marschner, M. Goethals, and M. Ercken, Variable-threshold resist models for lithography simulation, in Optical Microlithography XII, Proceedings of SPIE, Vol. 3679, 1999, pp. 176-182.

[38] A. E. Rosenbluth, S. Bukofsky, C. Fonseca, M. Hibbs, K. Lai, A. F. Molless, R. N. Singh, And A. K. K. Wong, Optimum mask and source patterns to print a given shape, J. Microlith. Microfab. Microsyst., 1 (2002), pp. 13-30.

[39] A. E. Rosenbluth, G. M. Gallatin, A. Lvov, R. L. Gordon, K. Lai, N. Seong, W. Hinsberg, J. Hoffnagle, F. Houle, And M. Sachez, Fast calculation of images for high numerical aperture lithography, in Optical/Laser Microlithography VIII, Proceedings of SPIE, Vol. 5377, 2004, pp. 615628.

[40] M. Rothschild, A roadmap for optical lithography, Opt. Photon. News, 21 (2010), pp. 26-31.

[41] L. Rudin, S. Osher, And E. Fatemi, Nonlinear total variation based noise removal algorithms, Phys. D, 60 (1992), pp. 259-268.

[42] Y. Shen, N. JiA, N. Wong, And E. Y. LAm, Robust level-set-based inverse lithography, Opt. Express, 19 (2011), pp. 5511-5521.

[43] Y. Shen, N. Wong, And E. Y. Lam, Level-set-based inverse lithography for photomask synthesis, Opt. Express, 17 (2009), pp. 23690-23701.

[44] Y. Shen, N. Wong, And E. Y. Lam, Aberration-aware robust mask design with level-set-based inverse lithography, in Photomask and Next-Generation Lithography Mask Technology XVII, Proceedings of SPIE, Vol. 7748, 2010, 77481U.

[45] S. Sherif, B. Saleh, And R. De LeOne, Binary image synthesis using mixed linear integer programming, IEEE Trans. Image Process., 4 (1995), pp. 1252-1257.

[46] S. Shiolri And H. TANABe, Fast optical proximity correction: Analytical method, in Optical/Laser Microlithography VIII, Proceedings of SPIE, Vol. 2440, 1995, pp. 261-269.

[47] A. K. Wong, Resolution Enhancement Techniques in Optical Lithography, SPIE Press, Bellingham, WA, 2001.

[48] J. YU AND P. YU, Impacts of cost functions on inverse lithography patterning, Opt. Express, 18 (2010), pp. 23331-23342.

Copyright (C) by SIAM. Unauthorized reproduction of this article is prohibited. 